\title{
A Scoping Review of the Association between Smartphone Use and Mental Health among College Students
}

\author{
Jeff Cain, EdD, MS ${ }^{1^{*}}$ D, Jordan L Kelley, PharmD, BCPS and Daniel Malcom, PharmD \\ ${ }^{1}$ Department of Pharmacy Practice \& Science, University of Kentucky College of Pharmacy, USA \\ 2University of Kentucky Healthcare Good Samaritan Hospital, USA
}

${ }^{3}$ Clinical and Administrative Sciences Department, Sullivan University College of Pharmacy \& Health

Sciences, USA

*Corresponding author: Jeff Cain, Department of Pharmacy Practice \& Science, University of Kentucky College of Pharmacy, 114M Lee T. Todd, Jr. Bldg, 789 South Limestone, Lexington, KY 40536-0596, USA, Tel: 859-230-2914

\begin{abstract}
A scoping review of the literature reporting on smartphone use and parameters of mental health from January 2008 to February 2018 was performed for the purpose of summarizing and describing current research findings regarding the association of smartphone use with anxiety, depression, stress, and sleep of college students. The study followed the Preferred Reporting Items for Systematic reviews and Meta-Analyses for Protocols 2015 (PRISMA-P 2015) guidelines.

Thirty-one articles met protocol criteria. Fifteen articles reported on studies of multiple mental healths related issues. Nineteen examined the association of smartphone use with anxiety, 19 examined depressions, eight examined sleep, and seven examined stress. Literature contained in this study indicated varied associations between problematic smartphone use and anxiety, depression, stress, and sleep. Consistent positive correlations were observed between problematic smartphone use, anxiety, stress and poor sleep. The correlations with depression were more disparate. The majority of studies were cross-sectional in nature and correlational. While existing research indicates correlations between problematic smartphone use and certain parameters of mental health, these associations should be studied further with research methodologies permitting more definitive conclusions regarding the links.
\end{abstract}

\section{Keywords}

Smartphones, Anxiety, Depression, Stress, Sleep, Mental health

\section{Introduction}

Since the Apple iPhone launch in 2008, prevalence of smartphone usage has reached near ubiquity with $81 \%$ of Americans reporting smartphone ownership in 2019. This proportion has more than doubled (from 35\%) since 2011, the first year the Pew Research Center reported figures on smartphone ownership [1]. Touted widely as one of the more influential innovations of the $21^{\text {st }}$ century, smartphones provide users with a host of capabilities including access to information, communication, and navigation, among countless others [2]. However, as with many other forms of technology, not all aspects of progress are positive. The negative effects associated with increased smartphone use are numerous and varied and include distraction of medical personnel [3], work-home interference [4], distracted driving [5], and academic distraction [6].

Recently, educators and researchers have expressed a growing concern that problematic smartphone use may also be contributing to growing mental health crisis among college students $[7,8]$. Some researchers and technology experts have hypothesized that design of smartphone devices themselves may exploit a variety of cognitive and psychological process that lead to an unhealthy attachment to the smartphones while simultaneously negatively impacting one's emotional state [9].

Research regarding the negative effects on mental health associated with smartphone use is relatively

Citation: Cain J, Kelley JL, Malcom D (2020) A Scoping Review of the Association between Smartphone Use and Mental Health among College Students. Int J Depress Anxiety 3:022. doi.org/10.23937/26434059/1710022

Accepted: October 05, 2020: Published: October 07, 2020

Copyright: (C) 2020 Cain J, et al. This is an open-access article distributed under the terms of the Creative Commons Attribution License, which permits unrestricted use, distribution, and reproduction in any medium, provided the original author and source are credited. 
new and covers a wide range of contributing factors; therefore a scoping review was determined to be the appropriate method to answer the research question. As opposed to a systematic review, which is guided by a highly-specific research question, a scoping review seeks to determine the broad range of evidence around a topic and summarize accordingly [10].

Scoping reviews are used to "examine the extent, range and nature of research activity", "summarize and disseminate research findings", and "identify research gaps in the existing literature" [11]. The purpose of this study was to describe current knowledge regarding the relationship between smartphone use of college students and aspects of their mental health. Specifically, this scoping review will answer the following questions: 1) What are the current research findings regarding the association of smartphone use with anxiety, depression, stress, and sleep of college students and, 2) What are the primary instruments used in studying the association between smartphone use and those parameters of mental health?

\section{Materials and Methods}

The protocol for this study followed the Preferred Reporting Items for Systematic reviews and Meta-Analyses for Protocols 2015 (PRISMA-P 2015) guidelines [12]. Although PRISMA-P was designed as pre-defined, quality assurance approach to conducting a systematic review, we followed these procedures (as applicable) in order to provide transparency of the methodological rigor of our scoping review process.

The search strategy was designed in collaboration with a medical librarian who performed the search. Re- levant studies to this review were identified through searches of four electronic databases (Medline, Web of Science, Scopus, and Psyclnfo). The searches were performed February 21-23, 2018 and were limited to articles published between January 1, 2008 and February 21,2018 . The initial year (2008) was chosen because it was the year following the initial release of the iPhone, which popularized smartphones.

The search used the following terms and Boolean operators: (Smartphone* OR iPhone* OR "smart phone" $O R$ "smart phones" OR "cell phone" OR "cell phones" OR "mobile phone" OR "mobile phones") AND (mental health OR addict" OR depress" OR anxiety OR Anxious OR stress* OR sleep OR nomophobia OR "fear of missing out" OR FOMO OR problematic) AND (College or University) AND Student ${ }^{*}$ ).

Rayyan, a web-based application designed to facilitate systematic reviews, was used for the initial screening of manuscripts [13]. All citations were imported into Rayyan and each researcher independently analyzed the titles and abstracts to determine if they met inclusion criteria. Articles were excluded when they were not research studies (eg, commentaries, review articles, etc.), did not include college students, were not from peer-reviewed journals, were published in a foreign language, or when their focus was on the use of smartphones for treatment or identification of mental health issues, as opposed to smartphones as a contributing factor to negative mental health outcomes. Figure 1 contains the PRISMA flow diagram for article selection. After the individual reviews, disagreements over the inclusion of particular articles were resolved by jointly re-reviewing those papers until consensus was reached.

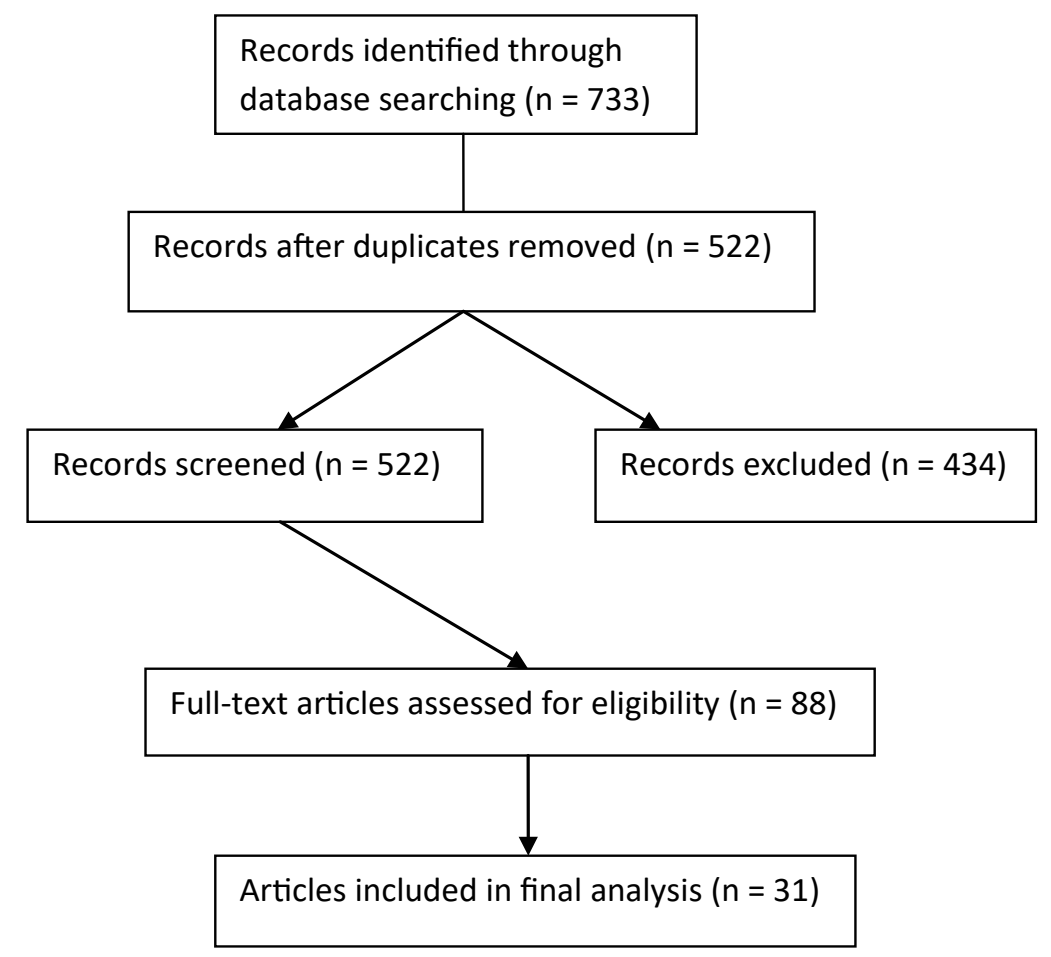

Figure 1: PRISMA flow diagram for article selection. 
Table 1: Studies included in scoping review and mental health aspect assessed $(N=31)$.

\begin{tabular}{|c|c|c|c|c|c|c|c|}
\hline \multirow[b]{2}{*}{ Author } & \multirow[b]{2}{*}{ Year } & \multirow[b]{2}{*}{ Country } & \multirow[b]{2}{*}{$\mathbf{N}$} & \multicolumn{4}{|c|}{ Mental health aspect } \\
\hline & & & & Anxiety & Depression & Sleep & Stress \\
\hline Alosaimi, et al. [14] & 2016 & Saudi Arabia & 2367 & & & $\mathrm{X}$ & \\
\hline Boumosleh, et al. [15] & 2017 & Lebanon & 688 & $x$ & $x$ & & \\
\hline Cheever, et al. [16] & 2014 & USA & 175 & $x$ & & & \\
\hline Chen, et al. [17] & 2016 & China & 1089 & $X$ & $x$ & & \\
\hline Chen, et al. [18] & 2017 & China & 1441 & $\mathrm{X}$ & $\mathrm{X}$ & $\mathrm{X}$ & \\
\hline Choi, et al. [19] & 2015 & South Korea & 448 & $x$ & $\mathrm{X}$ & & \\
\hline Deepali, et al. [20] & 2015 & India & 100 & & & $x$ & $x$ \\
\hline Demirci, et al. [21] & 2015 & Turkey & 319 & $x$ & $x$ & $\mathrm{X}$ & \\
\hline Elhai, et al. [22] & 2016 & USA & 68 & & $\mathrm{X}$ & & \\
\hline Eyvazlou, et al. [23] & 2016 & Iran & 450 & $\mathrm{X}$ & $x$ & $\mathrm{X}$ & \\
\hline Ezoe, et al. [24] & 2009 & Japan & 132 & & $x$ & & \\
\hline Gao, et al. [25] & 2018 & China & 1105 & $x$ & $x$ & & $x$ \\
\hline Gao, et al. [26] & 2017 & China & 722 & & $x$ & & \\
\hline Hawi, et al. [27] & 2017 & Lebanon & 341 & $x$ & & & \\
\hline Kim, et al. [28] & 2017 & South Korea & 200 & $x$ & $x$ & & \\
\hline Kim, et al. [29] & 2017 & South Korea & 608 & $\mathrm{X}$ & $\mathrm{X}$ & & $\mathrm{X}$ \\
\hline Kuang-Tsan, et al. [30] & 2017 & Taiwan & 332 & & & & $x$ \\
\hline Lee, et al. [31] & 2016 & South Korea & 1236 & $x$ & & & \\
\hline Lepp, et al. [32] & 2014 & USA & 536 & $x$ & & & \\
\hline Long, et al. [33] & 2016 & China & 1062 & $\mathrm{X}$ & $\mathrm{X}$ & & $\mathrm{X}$ \\
\hline Mok, et al. [34] & 2014 & South Korea & 448 & $x$ & $x$ & & \\
\hline Panova, et al. [35] & 2016 & USA & 318 & $\mathrm{X}$ & $\mathrm{X}$ & & \\
\hline Park, et al. [36] & 2012 & Korea & 319 & & $\mathrm{X}$ & & \\
\hline Rosen, et al. [37] & 2016 & USA & 734 & & & $\mathrm{X}$ & \\
\hline Saadat, et al. [38] & 2017 & Iran & 216 & $\mathrm{X}$ & $\mathrm{X}$ & & \\
\hline Sahin, et al. [39] & 2013 & Turkey & 576 & & & $\mathrm{X}$ & \\
\hline Samaha, et al. [40] & 2016 & Lebanon & 293 & & & & $x$ \\
\hline Sapacz, et al. [41] & 2016 & Canada & 152 & $x$ & & & \\
\hline Tao, et al. [42] & 2017 & China & 4747 & $x$ & $\mathrm{X}$ & $x$ & \\
\hline Tao, et al. [43] & 2017 & China & 2376 & & $x$ & & \\
\hline Wang, et al. [44] & 2015 & China & 600 & & & & $\mathrm{x}$ \\
\hline
\end{tabular}

\section{Results}

The initial database search resulted in 733 total records. Duplicate records $(N=211)$ were removed, resulting in 522 abstracts for initial screening. Abstract screening revealed that 434 abstracts did not meet inclusion criteria and resulted in 88 abstracts accessed for fulltext analysis. After a full-text analysis, 31 articles were determined to meet study criteria, shown in Table 1.

Articles that assessed multiple mental health aspects (eg, depression and anxiety) were counted within each category for analysis. Thus, the total number of studies adds up to more than 31 . Of those 31 articles, 19 (61.3\%) examined the association of smartphone use with anxiety (Table 2), 19 (61.3\%) examined depression (Table 3), eight (25.8\%) examined sleep (Table 4), and seven $(22.6 \%)$ examined stress (Table 5 ).

\section{Instruments measuring problematic smartphone use}

Problematic smartphone overuse can be measured in a variety of different ways depending on the assessment instrument and intent of the investigators. For example, overuse and dependence are two different types of problematic use. Twenty-six of the 31 studies in our analysis (83.9\%) employed a previously published scale to assess smartphone use or overuse, while five studies (16.1\%) [20,32,33,36,38] used an investigator-developed tool to assess smartphone use or dependence. Six instruments accounted for 15 of the 31 studies (48.4\%) analyzed. Kwon, et al. Smartphone Addiction Scale (SAS) [53] as well as its short version developed by the same research group (SAS-SV) [51] were the most commonly used assessment tools for smartphone use in the studies we analyzed at four and three 
Table 2: Studies evaluating link between smartphone use and anxiety $(\mathrm{N}=19)$.

\begin{tabular}{|c|c|c|c|}
\hline Author (s) & $\begin{array}{l}\text { Smartphone use } \\
\text { scale }\end{array}$ & Anxiety scale & Findings \\
\hline $\begin{array}{l}\text { Boumosleh, } \\
\text { et al. [15] }\end{array}$ & $\begin{array}{l}\text { Smartphone } \\
\text { addiction } \\
\text { inventory (SPAI) } \\
{[45]}\end{array}$ & $\begin{array}{l}\text { Generalized } \\
\text { anxiety disorder-7 } \\
(\text { GAD-7) [46] }\end{array}$ & $\begin{array}{l}\text { Anxiety was an independent positive predictive factor of smartphone } \\
\text { addiction. Anxiety (mean SPAI score: Anxious } 59.04 \mathrm{vs} \text {. non-anxious } \\
54.62, p=0.028 \text { ) The SPAI score was found to be significantly higher } \\
\text { for anxiety in a multiple linear regression with adjustment and the total } \\
\text { variance explained by the model was } 21 \% \text { for anxiety (Std. } B=0.122, p \\
=0.034 \text { ). }\end{array}$ \\
\hline $\begin{array}{l}\text { Cheever, et } \\
\text { al. [16] }\end{array}$ & $\begin{array}{l}\text { Wireless mobile } \\
\text { device (WMD) } \\
\text { use scale [47] }\end{array}$ & $\begin{array}{l}\text { State-trait anxiety } \\
\text { inventory (STAI) } \\
{[48]}\end{array}$ & $\begin{array}{l}\text { Anxiety was shown to increase over time in participants without their } \\
\text { devices regardless of its location }(p<0.01) \text {. Heavy users experienced } \\
\text { the most stress by the absence of the mobile device }(p=0.01) \text {. }\end{array}$ \\
\hline $\begin{array}{l}\text { Chen, et al. } \\
{[17]}\end{array}$ & $\begin{array}{l}\text { Mobile phone } \\
\text { addiction scale- } \\
\text { xiong (MPAS-X) } \\
{[49]}\end{array}$ & $\begin{array}{l}\text { Self- } \\
\text { consciousness } \\
\text { scale - social } \\
\text { anxiety subscale } \\
{[50]}\end{array}$ & $\begin{array}{l}\text { In a regression model, smartphone addiction was a significant predictor } \\
\text { of anxiety }(B=0.50, t=3.27, p<0.01) \text {. However, when the model } \\
\text { was controlled for interpersonal problems, the level of addiction to } \\
\text { smartphones had no significant effect on anxiety }(B=0.24, t=1.54, p> \\
0.05) \text {. }\end{array}$ \\
\hline $\begin{array}{l}\text { Chen, et al. } \\
\text { [18] }\end{array}$ & $\begin{array}{l}\text { Smartphone } \\
\text { addiction scale- } \\
\text { short version } \\
\text { (SAS-SV) [51] }\end{array}$ & $\begin{array}{l}\text { Self-rating } \\
\text { anxiety scale- } \\
\text { zung (SAS-Z) } \\
{[52]}\end{array}$ & $\begin{array}{l}\text { Smartphone addiction rate was } 29.8 \%(30.3 \% \text { in males, } 29.3 \% \text { in } \\
\text { females). In a multivariate logistic regression analysis, male students } \\
\text { with comorbid anxiety were more likely to have smartphone addiction } \\
(\mathrm{OR}=1.78,95 \% \mathrm{Cl} 1.09-2.89, \mathrm{p}<0.05) . \text { Female students with anxiety } \\
\text { were also more likely to have smartphone addiction }(\mathrm{OR}=2.31,95 \% \mathrm{Cl} \\
1.18-4.51, \mathrm{p}<0.05) .\end{array}$ \\
\hline $\begin{array}{l}\text { Choi, et al. } \\
\text { [19] }\end{array}$ & $\begin{array}{l}\text { Smartphone } \\
\text { addiction scale } \\
\text { (SAS) [53] }\end{array}$ & $\begin{array}{l}\text { State-trait anxiety } \\
\text { inventory (STAI) } \\
{[48]}\end{array}$ & $\begin{array}{l}\text { In a stepwise multiple regression analysis, higher anxiety was found to } \\
\text { be independently associated with higher levels of smartphone addiction } \\
(\text { Std. } B=0.224, t=4.426, p<0.01) \text {. }\end{array}$ \\
\hline $\begin{array}{l}\text { Demirci, et al. } \\
\text { [21] }\end{array}$ & $\begin{array}{l}\text { Smartphone } \\
\text { addiction scale } \\
\text { (SAS) [53] }\end{array}$ & $\begin{array}{l}\text { Beck anxiety } \\
\text { inventory (BAI) } \\
{[54]}\end{array}$ & $\begin{array}{l}\text { Smartphone addiction scores were significantly higher in females }(p< \\
0.01) \text { and anxiety }(p<0.01) \text {, depression }(p<0.01) \text {. Daytime dysfunction } \\
(p<0.01) \text { as measured by the PSQI score was higher in those that had } \\
\text { high use of smartphones compared to the low use group. In a linear } \\
\text { regression model, anxiety was significantly associated with smartphone } \\
\text { addiction severity (Std. } B=0.094, t=3.084, p<0.01) \text {. }\end{array}$ \\
\hline $\begin{array}{l}\text { Eyvazlou, et } \\
\text { al. [23] }\end{array}$ & $\begin{array}{l}\text { Internet over-use } \\
\text { scale and cell- } \\
\text { phone over-use } \\
\text { scale (IOS/COS) } \\
{[55]}\end{array}$ & $\begin{array}{l}\text { General health } \\
\text { questionnaire } \\
\text { 28-anxiety } \\
\text { and insomnia } \\
\text { subscale (GHQ- } \\
\text { 28) [56] }\end{array}$ & $\begin{array}{l}\text { A multivariate regression analysis investigating cell phone overuse } \\
\text { showed significant effects on social dysfunction }(p<0.05) \text { and } \\
\text { depression }(p=0.01) \text { but showed no significant impact on anxiety }(p> \\
0.05) \text {. However, this study did look at sleep quality and found that effects } \\
\text { on sleep quality also had an impact on the anxiety and depression } \\
\text { scales }(p<0.01) \text {. }\end{array}$ \\
\hline $\begin{array}{l}\text { Gao, et al. } \\
\text { [25] }\end{array}$ & $\begin{array}{l}\text { Mobile phone } \\
\text { addiction index } \\
\text { (MPAI) [57] }\end{array}$ & $\begin{array}{l}\text { Depression } \\
\text { anxiety and } \\
\text { stress scale } \\
\text { (DASS-21) [58] }\end{array}$ & $\begin{array}{l}\text { Participants were more likely to have alexithymia (inability to identify } \\
\text { or show feelings) which affected mobile phone addiction }(p<0.01) \text {, } \\
\text { depression }(p<0.01) \text {, stress }(p<0.01) \text {, and anxiety }(p<0.01) \text {. } \\
\text { Depression and anxiety significantly affected mobile phone addiction ( } p \\
<0.01) \text {. Inclusion of anxiety in the model increased the explanation of } \\
\text { mobile phone addiction by } 10.3 \% \text {. }\end{array}$ \\
\hline $\begin{array}{l}\text { Hawi, et al. } \\
\text { [27] }\end{array}$ & $\begin{array}{l}\text { Smartphone } \\
\text { addiction scale } \\
\text { (SAS) [53] }\end{array}$ & $\begin{array}{l}\text { Beck anxiety } \\
\text { inventory (BAl) } \\
{[54]}\end{array}$ & $\begin{array}{l}\text { The odds of having anxiety in undergraduate students addicted to their } \\
\text { smartphones was } 4.7 \text { times higher than non-addicted students }(95 \% \mathrm{Cl} \\
1.511-14.659, \mathrm{p}=0.008) \text {. }\end{array}$ \\
\hline $\begin{array}{l}\text { Kim, et al. } \\
\text { [28] }\end{array}$ & $\begin{array}{l}\text { Smartphone } \\
\text { addiction } \\
\text { proneness scale } \\
\text { (SAPS) [59] }\end{array}$ & $\begin{array}{l}\text { Experiences } \\
\text { in close } \\
\text { relationships- } \\
\text { revised-Korean } \\
\text { [60] }\end{array}$ & $\begin{array}{l}\text { The path from attachment anxiety to loneliness was significant }(B= \\
0.55, t=5.99) \text {. The total effect of attachment avoidance on smartphone } \\
\text { addiction was significant }(p<0.01) \text {. The total effect of attachment } \\
\text { anxiety and smartphone addiction was also significant }(p<0.01) \text {. }\end{array}$ \\
\hline $\begin{array}{l}\text { Kim, et al. } \\
\text { [29] }\end{array}$ & $\begin{array}{l}\text { Smartphone } \\
\text { addiction } \\
\text { proneness scale } \\
\text { (SAPS) [59] }\end{array}$ & $\begin{array}{l}\text { Investigator- } \\
\text { developed }\end{array}$ & $\begin{array}{l}\text { Smartphone overuse was associated with psychotic anxiety by a twofold } \\
\text { increase }(p<0.05) \text { when compared to those with psychological anxiety. } \\
\text { Students who reported overuse of smartphones were more likely to } \\
\text { report their health was "not good" (OR } 1.98,95 \% \text { Cl } 1.22-3.21) \text {. }\end{array}$ \\
\hline $\begin{array}{l}\text { Lee, et al. } \\
\text { [31] }\end{array}$ & $\begin{array}{l}\text { Questionnaire on } \\
\text { mobile } \\
\text { telephone } \\
\text { dependency [61] }\end{array}$ & $\begin{array}{l}\text { Self-Rating } \\
\text { anxiety scale- } \\
\text { zung (SAS-Z) } \\
{[52]}\end{array}$ & $\begin{array}{l}\text { The amount of time on smartphones and the purpose of use affected } \\
\text { dependency upon smartphones in both men and women. Daily use time } \\
\text { and dependency were directly correlated. Smartphone dependency was } \\
\text { directly correlated with risk of abnormal anxiety in men and women and } \\
\text { increased by } 10.1 \% \text { and } 9.2 \% \text {, respectively }(p<0.01) \text {. }\end{array}$ \\
\hline
\end{tabular}




\begin{tabular}{|c|c|c|c|}
\hline $\begin{array}{l}\text { Lepp, et al. } \\
\text { [32] }\end{array}$ & $\begin{array}{l}\text { Investigator- } \\
\text { developed }\end{array}$ & $\begin{array}{l}\text { Beck anxiety } \\
\text { inventory } \\
\text { (BAI) [54] }\end{array}$ & $\begin{array}{l}\text { The median cell phone use per day was } 278 \text { minutes with a median } \\
\text { of } 76 \text { texts sent per day. In a regression analysis, cell phone use was } \\
\text { positively related to anxiety }(B=0.10, p<0.05) \text {. }\end{array}$ \\
\hline $\begin{array}{l}\text { Long, et al. } \\
\text { [33] }\end{array}$ & $\begin{array}{l}\text { Problematic } \\
\text { cellular phone } \\
\text { use } \\
\text { questionnaire } \\
\text { (PCPUQ) [62] }\end{array}$ & $\begin{array}{l}\text { Self-rating } \\
\text { anxiety } \\
\text { scale-zung } \\
\text { (SAS-Z) [52] }\end{array}$ & $\begin{array}{l}\text { Anxiety and depression were collapsed into a single variable "emotional } \\
\text { symptoms". Through regressional analysis, emotional symptoms were } \\
\text { identified as a risk factor for problematic use }(p<0.05) \text {. }\end{array}$ \\
\hline $\begin{array}{l}\text { Mok, et al. } \\
\text { [34] }\end{array}$ & $\begin{array}{l}\text { Smartphone } \\
\text { addiction scale } \\
\text { (SAS) [53] }\end{array}$ & $\begin{array}{l}\text { State-trait anxiety } \\
\text { inventory (STAI) } \\
{[48]}\end{array}$ & $\begin{array}{l}\text { Mean anxiety scores were significantly higher in female respondents } \\
(45.69 \pm 9.42) v s \text {. male respondents }(41.60 \pm 9.68) \text {, which was } \\
\text { significant }(p<0.01) \text {. Smartphone addiction scores were also higher } \\
\text { in female respondents }(74.67 \pm 25.50) \text { vs. male respondents }(59.65 \\
\pm 21.08) \text {, which was significant }(p<0.01) \text {. In latent class analysis, } \\
\text { smartphone addiction was significantly accompanied by higher levels } \\
\text { of anxiety }(F=22.55, p<0.01) \text {. A common trend for psychosocial trait } \\
\text { factors was found for both sexes: Anxiety levels and neurotic personality } \\
\text { traits increased with addiction severity levels }(p<0.01) \text {. }\end{array}$ \\
\hline $\begin{array}{l}\text { Panova, et al. } \\
\text { [35] }\end{array}$ & $\begin{array}{l}\text { Questionnaire } \\
\text { about } \\
\text { experiences } \\
\text { related to the } \\
\text { internet (CERI) } \\
\text { [63] }\end{array}$ & $\begin{array}{l}\text { Mood and anxiety } \\
\text { symptom } \\
\text { questionnaire- } \\
\text { anxious arousal } \\
\text { subscale (MASQ) } \\
{[64]}\end{array}$ & $\begin{array}{l}\text { Correlation found between maladaptive mobile phone use and anxiety } \\
(r=0.452, p<0.01) \text {. Study suggests that long term use of mobile phone } \\
\text { use as a coping strategy may have a negative impact on mental health. }\end{array}$ \\
\hline $\begin{array}{l}\text { Saadat, et al. } \\
\text { [38] }\end{array}$ & $\begin{array}{l}\text { Investigator- } \\
\text { developed }\end{array}$ & $\begin{array}{l}\text { Beck anxiety } \\
\text { inventory } \\
\text { (BAI) [54] }\end{array}$ & $\begin{array}{l}\text { A significant correlation was found between mobile phone dependency } \\
\text { and anxiety scores }(r=0.190, p=0.005) \text {. }\end{array}$ \\
\hline $\begin{array}{l}\text { Sapacz, et al. } \\
\text { [41] }\end{array}$ & $\begin{array}{l}\text { Mobile phone } \\
\text { problem use } \\
\text { scale } \\
\text { (PMPU) [65] }\end{array}$ & $\begin{array}{l}\text { State-trait anxiety } \\
\text { inventory (STAI) } \\
\text { [48] } \\
\text { Self-beliefs } \\
\text { related to social } \\
\text { anxiety scale } \\
\text { (SANX) [66] }\end{array}$ & $\begin{array}{l}\text { In a regression analysis, problematic mobile phone use did not predict } \\
\text { state anxiety for any of the experimental conditions, including "taken } \\
\text { away" }(B=-0.010, t=-0.200, p=0.842) \text {, "salient" }(B=-0.025, t= \\
-0.521, p=0.606 \text {, and "hidden" ( } B=-0.045, t=-0.668, p=0.509)\end{array}$ \\
\hline $\begin{array}{l}\text { Tao, et al. } \\
{[42]}\end{array}$ & $\begin{array}{l}\text { Self-rating } \\
\text { questionnaire } \\
\text { for adolescent } \\
\text { problematic } \\
\text { mobile phone use } \\
\text { (SQAPMPU) [67] }\end{array}$ & $\begin{array}{l}\text { Self-rating } \\
\text { anxiety scale- } \\
\text { zung (SAS-Z) } \\
{[52]}\end{array}$ & $\begin{array}{l}\text { Higher rates of anxiety symptoms were seen in those with problematic } \\
\text { mobile phone use }(p<0.01) \text {. Higher rates of psychopathological } \\
\text { symptoms, depression, and anxiety symptoms were seen in those with } \\
\text { internet addiction }(p<0.01) \text { and poor sleep quality }(p<0.01) \text {. }\end{array}$ \\
\hline
\end{tabular}

Table 3: Studies evaluating link between smartphone use and depression ( $N=19)$.

\begin{tabular}{|c|c|c|c|}
\hline Author (s) & $\begin{array}{l}\text { Smartphone } \\
\text { use scale }\end{array}$ & Depression scale & Findings \\
\hline $\begin{array}{l}\text { Boumosleh, } \\
\text { et al. [15] }\end{array}$ & $\begin{array}{l}\text { Smartphone } \\
\text { addiction } \\
\text { inventory (SPAI) } \\
{[45]}\end{array}$ & $\begin{array}{l}\text { Patient health } \\
\text { questionnaire-9 } \\
\text { (PHQ-9) [68] }\end{array}$ & $\begin{array}{l}\text { Depression was an independent positive predictive factor of } \\
\text { smartphone addiction after adjustment for potential confounders. A } \\
\text { multiple linear regression with adjustment for age, personality type, } \\
\text { year in program, age at first smartphone use, duration of smartphone } \\
\text { use, and use a smartphone for calling family members, entertainment, } \\
\text { or other purposes showed the total variance explained by the model } \\
\text { was } 23 \% \text { for depression (Std. } B=0.201, p<0.01 \text { ). }\end{array}$ \\
\hline $\begin{array}{l}\text { Chen, et al. } \\
\text { [17] }\end{array}$ & $\begin{array}{l}\text { Mobile phone } \\
\text { addiction } \\
\text { scale-xiong } \\
\text { (MPAS-X) [49] }\end{array}$ & $\begin{array}{l}\text { Center for } \\
\text { epidemiologic studies } \\
\text { depression scale } \\
\text { (CES-D) [69] }\end{array}$ & $\begin{array}{l}\text { In the depression regression model, } 45 \% \text { of the variance in } \\
\text { depression is accounted for by the mobile phone addiction (MPA) } \\
\text { level }(p<0.01) . \text { MPA addiction level was a significant predictor of } \\
\text { depression }(B=0.43, t=3.15, p<0.01) .33 \% \text { of the variance in } \\
\text { interpersonal problems could be accounted for by the MPA level, } \\
\text { and the MPA level significantly predicted interpersonal problems (B = } \\
0.49, t=3.30, p<0.01) \text {. }\end{array}$ \\
\hline $\begin{array}{l}\text { Chen, et al. } \\
\text { [18] }\end{array}$ & $\begin{array}{l}\text { Smartphone } \\
\text { addiction scale- } \\
\text { short version } \\
\text { (SAS-SV) [51] }\end{array}$ & $\begin{array}{l}\text { Center for } \\
\text { epidemiologic studies } \\
\text { depression scale } \\
\text { (CES-D) [69] }\end{array}$ & $\begin{array}{l}\text { Overall smartphone addiction rate was } 29.8 \%(30.3 \% \text { in males, } \\
29.3 \% \text { in females). In a multivariate logistic regression analysis, male } \\
\text { students with depression symptoms were not significantly more likely } \\
\text { to have smartphone addiction, while female students with depression } \\
\text { symptoms were more likely to have smartphone addiction }(\mathrm{OR}=1.84 \text {, } \\
95 \% \mathrm{Cl} 1.21-2.79, \mathrm{p}<0.01) \text {. }\end{array}$ \\
\hline
\end{tabular}




\begin{tabular}{|c|c|c|c|}
\hline $\begin{array}{l}\text { Choi, et al. } \\
{[19]}\end{array}$ & $\begin{array}{l}\text { Smartphone } \\
\text { addiction } \\
\text { scale (SAS) [53] }\end{array}$ & $\begin{array}{l}\text { Beck depression } \\
\text { inventory (BDI) [70] }\end{array}$ & $\begin{array}{l}\text { In a stepwise multiple regression analysis, higher depression scores } \\
\text { were found to be protective against smartphone addiction, meaning } \\
\text { lower smartphone addiction scores (Std. } B=-0.215, t=-3.598, p< \\
0.01 \text { ). }\end{array}$ \\
\hline $\begin{array}{l}\text { Demirci, et } \\
\text { al. [21] }\end{array}$ & $\begin{array}{l}\text { Smartphone } \\
\text { addiction } \\
\text { scale (SAS) [53] }\end{array}$ & $\begin{array}{l}\text { Beck depression } \\
\text { inventory (BDI) [70] }\end{array}$ & $\begin{array}{l}\text { Smartphone use severity was positively correlated with depression } \\
\text { scores }(r=0.276, p<0.001) \text {. Significantly more high smartphone } \\
\text { users had depression than low smartphone users }(p=0.005) . \text { In } \\
\text { a stepwise linear regression model, depression was significantly } \\
\text { associated with smartphone addiction severity (Std. } B=0.067, t= \\
2.069, p=0.040) \text {. }\end{array}$ \\
\hline $\begin{array}{l}\text { Elhai, et al. } \\
\text { [22] }\end{array}$ & $\begin{array}{l}\text { Smartphone } \\
\text { addiction scale- } \\
\text { short version } \\
\text { (SAS-SV) [51] }\end{array}$ & $\begin{array}{l}\text { Patient health } \\
\text { questionnaire-9 } \\
\text { (PHQ-9) [68] }\end{array}$ & $\begin{array}{l}\text { Smartphone use minutes were significantly correlated with } \\
\text { problematic smartphone use }(p=0.01) \text {. Depression severity was not } \\
\text { related to objective smartphone use }(B=36.53, p=0.43) \text { and the } \\
\text { slope in the growth curve model showed and inverse relationship } \\
\text { between objective smartphone use and initial depression. Therefore, } \\
\text { higher baseline depression severity was associated with decreased } \\
\text { use over the week. }\end{array}$ \\
\hline $\begin{array}{l}\text { Eyvazlou, et } \\
\text { al. [23] }\end{array}$ & $\begin{array}{l}\text { Internet over-use } \\
\text { scale and cell- } \\
\text { phone over-use } \\
\text { scale (IOS/COS) } \\
{[55]}\end{array}$ & $\begin{array}{l}\text { General health } \\
\text { questionnaire } \\
\text { 28-severe } \\
\text { depression subscale } \\
(\mathrm{GHQ}-28)[56]\end{array}$ & $\begin{array}{l}\text { Lowest scores of general health status were from females }(16.83 \pm \\
12.45) \text { and fourth-year occupational health students }(9.82 \pm 7.86) \\
\text { smartphone overuse had a significant effect on depression subscales } \\
(p<0.01) .\end{array}$ \\
\hline $\begin{array}{l}\text { Ezoe, et al. } \\
{[24]}\end{array}$ & $\begin{array}{l}\text { Mobile phone } \\
\text { dependence } \\
\text { questionnaire } \\
\text { (MPDQ) [71] }\end{array}$ & $\begin{array}{l}\text { Self-rating } \\
\text { depression scale } \\
\text { (SDS) }[72]\end{array}$ & $\begin{array}{l}\text { There was a positive small but not significant correlation between } \\
\text { mobile phone dependence and depression scores }(r=0.12, p>0.05) \text {. }\end{array}$ \\
\hline $\begin{array}{l}\text { Gao, et al. } \\
{[25]}\end{array}$ & $\begin{array}{l}\text { Mobile phone } \\
\text { addiction index } \\
\text { (MPAI) [57] }\end{array}$ & $\begin{array}{l}\text { Depression anxiety } \\
\text { and stress scale } \\
\text { (DASS-21) [58] }\end{array}$ & $\begin{array}{l}\text { Alexithymia affected depression }(B=0.503, t=19.311, p<0.01) \text { and } \\
\text { the mediating effect was } 62 \% \text {. In the regression model, depression } \\
\text { significantly affected mobile phone addiction }(B=0.407, t=14.799, p \\
<0.001) \text {. Inclusion of anxiety in the model increased the explanation } \\
\text { of mobile phone addiction by } 7.8 \% \text {. The regression showed that } \\
\text { depression was a predictor of mobile phone addiction due to inability } \\
\text { to control craving ( } p<0.01) \text {, feeling anxious and lost }(p<0.01) \text {, } \\
\text { withdrawal or escape }(p<0.01) \text {, and productivity loss }(p<0.01) \text {. }\end{array}$ \\
\hline $\begin{array}{l}\text { Gao, et al. } \\
{[26]}\end{array}$ & $\begin{array}{l}\text { Mobile phone } \\
\text { addiction scale- } \\
\text { hong (MPAS-H) } \\
\text { [73] }\end{array}$ & $\begin{array}{l}\text { Beck depression } \\
\text { inventory-II (BDI-II) } \\
{[74]}\end{array}$ & $\begin{array}{l}\text { Smartphone addiction and depression were positively correlated ( } p \\
<0.05 \text { ) among participants. In a structural equation model, there } \\
\text { were indirect relationships of neuroticism on quality of life through } \\
\text { smartphone addiction. The total effect of smartphone addiction and } \\
\text { depression was }-2.171 \text { based on direct effect }(25 \%) \text {, sole mediation of } \\
\text { depression ( } 39 \%) \text {, sole mediation of smartphone addiction }(29 \%) \text {, and } \\
\text { the continuous path of smartphone addiction and depression }(41 \%) \text {. }\end{array}$ \\
\hline $\begin{array}{l}\text { Kim, et al. } \\
{[28]}\end{array}$ & $\begin{array}{l}\text { Smartphone } \\
\text { addiction } \\
\text { proneness scale } \\
\text { (SAPS) [59] }\end{array}$ & $\begin{array}{l}\text { Center for } \\
\text { epidemiologic studies } \\
\text { depression scale } \\
\text { (CES-D) [69] }\end{array}$ & $\begin{array}{l}\text { The paths from loneliness to depression }(B=0.54, t=2.46) \text { and from } \\
\text { depression to smartphone addiction }(B=0.34, t=3.06) \text { were valid } \\
\text { paths of significance in the effect analysis. Depression's mediating } \\
\text { effect with attachment avoidance }(p<0.05) \text { and attachment anxiety } \\
(p<0.05) \text { were significant. High attachment anxiety, loneliness, and } \\
\text { depression predicted smartphone addiction. }\end{array}$ \\
\hline $\begin{array}{l}\text { Kim, et al. } \\
\text { [29] }\end{array}$ & $\begin{array}{l}\text { Smartphone } \\
\text { addiction } \\
\text { proneness scale } \\
\text { (SAPS) [59] }\end{array}$ & $\begin{array}{l}\text { Investigator- } \\
\text { developed }\end{array}$ & $\begin{array}{l}\text { Perceived psychological health, such as stress and depression, } \\
\text { closely related to smartphone overuse }(p<0.05) \text {. Participants that } \\
\text { had symptoms of depression were } 1.91 \text { times more likely to overuse } \\
\text { smartphones }(95 \% \mathrm{Cl} 1.27-2.86, p=0.0018) \text {. }\end{array}$ \\
\hline $\begin{array}{l}\text { Long, et al. } \\
\text { [33] }\end{array}$ & $\begin{array}{l}\text { Problematic } \\
\text { cellular } \\
\text { phone use } \\
\text { questionnaire } \\
\text { (PCPUQ) [62] }\end{array}$ & $\begin{array}{l}\text { Self-rating } \\
\text { depression scale } \\
\text { (SDS) [72] }\end{array}$ & $\begin{array}{l}\text { Problematic cell phone use was associated with hours of use }(p< \\
0.01) \text {, frequency of mobile phone change }(p<0.01) \text {, and monthly } \\
\text { smartphone bill }(p<0.01) \text {. There was no difference in usage } \\
\text { preference between problematic versus non problematic users ( } p \\
=0.18) \text {. The risk factors for problematic smartphone use included } \\
\text { depression as measured by the SDS }(p<0.01) \text {. }\end{array}$ \\
\hline $\begin{array}{l}\text { Mok, et al. } \\
{[34]}\end{array}$ & $\begin{array}{l}\text { Smartphone } \\
\text { addiction } \\
\text { scale (SAS) [53] }\end{array}$ & $\begin{array}{l}\text { Beck depression } \\
\text { inventory (BDI) [70] }\end{array}$ & $\begin{array}{l}\text { Mean depression scores were significantly higher in female } \\
\text { respondents }(19.58 \pm 13.40) \text { vs. male respondents }(15.58 \pm 10.75) \text {, } \\
\text { which was significant }(p<0.01) \text {. Smartphone addiction scores } \\
\text { were also higher in female respondents }(74.67 \pm 25.50) v s \text {. male } \\
\text { respondents }(59.65 \pm 21.08) \text {, which was significant }(p<0.01) \text {. In } \\
\text { latent class analysis, smartphone addiction was not a significant } \\
\text { factor in differentiating levels of depressive feelings }(F=0.275, p= \\
0.76) \text {. }\end{array}$ \\
\hline
\end{tabular}




\begin{tabular}{|c|c|c|c|}
\hline $\begin{array}{l}\text { Panova, et } \\
\text { al. [35] }\end{array}$ & $\begin{array}{l}\text { Questionnaire } \\
\text { about } \\
\text { experiences } \\
\text { related to the } \\
\text { internet (CERI) } \\
\text { [75] }\end{array}$ & $\begin{array}{l}\text { Mood and } \\
\text { anxiety symptom } \\
\text { questionnaire- } \\
\text { anhedonic } \\
\text { depression subscale } \\
\text { (MASQ) [64] }\end{array}$ & $\begin{array}{l}\text { A significant correlation was found between maladaptive mobile } \\
\text { phone use and depression }(r=0.194, p<0.01)\end{array}$ \\
\hline $\begin{array}{l}\text { Park, et al. } \\
{[36]}\end{array}$ & $\begin{array}{l}\text { Investigator- } \\
\text { developed }\end{array}$ & $\begin{array}{l}\text { Center for } \\
\text { epidemiologic studies } \\
\text { depression scale } \\
\text { (CES-D) [69] }\end{array}$ & $\begin{array}{l}\text { Respondents who used smartphones for bonding and bridging ties as } \\
\text { well as for sharing a sense of support were likely to have lower levels } \\
\text { of depression. Bonding social ties were positively related to perceived } \\
\text { social support }(r=0.44, p<0.001) \text {. }\end{array}$ \\
\hline $\begin{array}{l}\text { Saadat, et al. } \\
\text { [38] }\end{array}$ & $\begin{array}{l}\text { Investigator- } \\
\text { developed }\end{array}$ & $\begin{array}{l}\text { Beck depression } \\
\text { inventory (BDI) }[70]\end{array}$ & $\begin{array}{l}\text { A significant correlation was found between mobile phone } \\
\text { dependency and depression scores }(r=0.295, p=0.000) \text {. }\end{array}$ \\
\hline $\begin{array}{l}\text { Tao, et al. } \\
{[43]}\end{array}$ & $\begin{array}{l}\text { Self-rating } \\
\text { questionnaire } \\
\text { for adolescent } \\
\text { problematic } \\
\text { mobile phone } \\
\text { use (SQAPMPU) } \\
\text { [67] }\end{array}$ & $\begin{array}{l}\text { Self-rating } \\
\text { depression scale } \\
\text { (SDS) [72] }\end{array}$ & $\begin{array}{l}\text { Prevalence of problematic mobile phone use was } 27.9 \% \text {, while } \\
\text { prevalence of depressive symptoms was } 18.9 \% \text { and alcohol use was } \\
37.5 \% \text {. There was no significant increase in alcohol use in depressed } \\
\text { students (OR } 1.183,95 \% \mathrm{Cl} 0.931-1.502) \text {. Problematic mobile phone } \\
\text { use was independently associated with alcohol use (OR } 1.295,95 \% \\
\mathrm{Cl} 1.040-1.611) \text {. In a multivariate regression analysis, there was } \\
\text { an additive interaction between problematic use and depressive } \\
\text { symptoms with alcohol use (OR } 1.456,95 \% \mathrm{Cl} 1.044-2.030, \mathrm{p}= \\
0.027 \text { ). }\end{array}$ \\
\hline $\begin{array}{l}\text { Tao, et al. } \\
{[42]}\end{array}$ & $\begin{array}{l}\text { Self-rating } \\
\text { questionnaire } \\
\text { for adolescent } \\
\text { problematic } \\
\text { mobile phone } \\
\text { use (SQAPMPU) } \\
\text { [67] }\end{array}$ & $\begin{array}{l}\text { Center for } \\
\text { epidemiologic studies } \\
\text { depression scale } \\
\text { (CES-D) [69] }\end{array}$ & $\begin{array}{l}\text { Problematic mobile phone use and poor sleep quality were observed } \\
\text { in } 28.1 \% \text { and } 9.8 \% \text { of participants, respectively. Depression } \\
\text { symptoms were significantly higher in students with problematic } \\
\text { mobile phone use }(p<0.01)\end{array}$ \\
\hline
\end{tabular}

Table 4: Studies evaluating the link between smartphone use and sleep $(N=8)$.

\begin{tabular}{|c|c|c|c|}
\hline Author(s) & $\begin{array}{l}\text { Smartphone use } \\
\text { scale }\end{array}$ & $\begin{array}{l}\text { Sleep quality } \\
\text { scale }\end{array}$ & Findings \\
\hline $\begin{array}{l}\text { Alosaimi, et } \\
\text { al. [14] }\end{array}$ & $\begin{array}{l}\text { Problematic use of } \\
\text { mobile } \\
\text { phones (PUMP) } \\
\text { scale [76] }\end{array}$ & $\begin{array}{l}\text { Investigator- } \\
\text { developed }\end{array}$ & $\begin{array}{l}23.4 \% \text { of the subjects rated themselves as addicted to their smartphones } \\
\text { and } 43 \% \text { reported decreased sleep. Decreased sleeping hours as a } \\
\text { consequence of smartphone use was significantly correlated }(r=0.379) \\
\text { with problematic smartphone use score. }\end{array}$ \\
\hline $\begin{array}{l}\text { Chen, et al. } \\
\text { [18] }\end{array}$ & $\begin{array}{l}\text { Smartphone } \\
\text { addiction scale- } \\
\text { short version } \\
\text { (SAS-SV) [51] }\end{array}$ & $\begin{array}{l}\text { Pittsburgh } \\
\text { sleep quality } \\
\text { index (PSQI) } \\
{[77]}\end{array}$ & $\begin{array}{l}\text { Smartphone addiction rate was } 29.8 \%(30.3 \% \text { in males, } 29.3 \% \text { in females). } \\
\text { In a multivariate logistic regression analysis, male students with comorbid } \\
\text { sleep problems were } 3 x \text { more likely to have a smartphone addiction (OR } \\
=3.19,95 \% \mathrm{Cl} 2.23-4.58, \mathrm{p}<0.01) \text {. Female students with sleep problems } \\
\text { were also more likely to have smartphone addiction (OR }=2.12,95 \% \mathrm{Cl} \\
1.50- \\
2.99, \mathrm{p}<0.01) \text {. }\end{array}$ \\
\hline $\begin{array}{l}\text { Deepali, et } \\
\text { al. [20] }\end{array}$ & $\begin{array}{l}\text { Investigator- } \\
\text { developed }\end{array}$ & $\begin{array}{l}\text { Developed } \\
\text { Pittsburgh } \\
\text { sleep quality } \\
\text { index (PSQI) } \\
{[77]}\end{array}$ & $\begin{array}{l}82.5 \% \text { of students who used their smartphones for more than two hours } \\
\text { each day had PSQI scores }>5 \text {, while only } 58.1 \% \text { of students who used } \\
\text { their devices for less than } 2 \text { hours per day had PSQI scores }>5 \text {. Increased } \\
\text { use of smartphones (more than two hours per day) was associated with } \\
\text { higher sleep deprivation }(p<0.01) \text { and increased sleep latency }(p<0.01) \text {. }\end{array}$ \\
\hline $\begin{array}{l}\text { Demirci, et } \\
\text { al. [21] }\end{array}$ & $\begin{array}{l}\text { Smartphone } \\
\text { addiction scale } \\
\text { (SAS) [53] }\end{array}$ & $\begin{array}{l}\text { Pittsburgh } \\
\text { sleep quality } \\
\text { index (PSQI) } \\
{[77]}\end{array}$ & $\begin{array}{l}\text { Smartphone use disorder severity was positively correlated with overall } \\
\text { PSQI scores }(r=0.156, p<0.05) \text {, as well as subjective sleep quality }(r \\
=0.138, p<0.05) \text {, sleep disturbance }(r=0.153, p<0.05) \text {, and daytime } \\
\text { dysfunction }(r=0.244, p<0.01) \text {. However, in a stepwise linear regression, } \\
\text { there was no direct effect of high smartphone use on sleep quality (Std. } B= \\
-0.022, t=-0.379, p=0.705) \text {. }\end{array}$ \\
\hline $\begin{array}{l}\text { Eyvazlou, } \\
\text { et al. [23] }\end{array}$ & $\begin{array}{l}\text { Cell phone } \\
\text { overuse scale } \\
\text { (COPS) [78] }\end{array}$ & $\begin{array}{l}\text { Pittsburgh } \\
\text { sleep quality } \\
\text { index (PSQI) } \\
{[77]}\end{array}$ & $\begin{array}{l}\text { Smartphone overuse had a significant relationship with quality of sleep } \\
\text { focusing on sleep latency }(p<0.01) \text {, sleep disturbance }(p<0.01) \text {, daytime } \\
\text { dysfunction }(p<0.01) \text {, and use of sleep medication }(p<0.01) \text {. Multiple } \\
\text { regression analysis showed that overuse affected quality of sleep ( } p \\
<0.01) \text {, but subjective sleep duration and quality was not significant. } \\
\text { Adjusted PSQI showed a significant relationship between sleep problems } \\
\text { and smartphone overuse }(r 2=0.181, p<0.01) \text {. Students' general health } \\
\text { had a meaningful relationship with their quality of sleep }(p<0.01) \text {, which } \\
\text { also affected anxiety and depression scales. }\end{array}$ \\
\hline
\end{tabular}




\begin{tabular}{|c|c|c|c|}
\hline $\begin{array}{l}\text { Rosen, et } \\
\text { al. [37] }\end{array}$ & $\begin{array}{l}\text { Media and } \\
\text { technology usage } \\
\text { and attitudes scale } \\
\text { (Daily smartphone } \\
\text { usage subscale) } \\
\text { [47] }\end{array}$ & $\begin{array}{l}\text { Medical } \\
\text { outcomes } \\
\text { study } \\
\text { sleep measure } \\
{[79]}\end{array}$ & $\begin{array}{l}50 \% \text { of participants kept their phones close at night and } 49 \% \text { admitted to } \\
\text { checking it for something other than time. } 33.5 \% \text { of participants reported } \\
\text { getting less than } 7 \text { hours of sleep per night and } 32 \% \text { getting exactly } \\
7 \text { hours. Participants that awakened to check their phones showed } \\
\text { significantly more problems sleeping }(p<0.01) \text {. }\end{array}$ \\
\hline $\begin{array}{l}\text { Sahin, et } \\
\text { al. [39] }\end{array}$ & $\begin{array}{l}\text { Problematic mobile } \\
\text { phone use (PMPU) } \\
\text { scale [65] }\end{array}$ & $\begin{array}{l}\text { Pittsburgh } \\
\text { sleep quality } \\
\text { index (PSQI) } \\
{[77]}\end{array}$ & $\begin{array}{l}\text { Problematic smartphone use scores and sleep quality scores showed a } \\
\text { positive correlation }(r=-0.297 ; p<0.01) \text {. The quality of sleep deteriorated } \\
\text { as dependence on mobile phone increased. }\end{array}$ \\
\hline $\begin{array}{l}\text { Tao, et al. } \\
\text { [42] }\end{array}$ & $\begin{array}{l}\text { Self-rating } \\
\text { questionnaire } \\
\text { for adolescent } \\
\text { problematic } \\
\text { mobile phone use } \\
\text { (SQAPMPU) [67] }\end{array}$ & $\begin{array}{l}\text { Pittsburgh } \\
\text { sleep quality } \\
\text { index (PSQI) } \\
{[77]}\end{array}$ & $\begin{array}{l}\text { Problematic smartphone use and poor sleep quality were observed in } \\
28.1 \% \text { and } 9.8 \% \text { of participants, respectively. Logistic regression models } \\
\text { suggested indicated independent associations of problematic smartphone } \\
\text { use and sleep quality with mental health }(p<0.001) \text {. }\end{array}$ \\
\hline
\end{tabular}

Table 5: Studies evaluating the link between smartphone use and stress $(N=7)$.

\begin{tabular}{|c|c|c|c|}
\hline Author(s) & $\begin{array}{l}\text { Smartphone use } \\
\text { scale }\end{array}$ & Stress scale & Findings \\
\hline $\begin{array}{l}\text { Deepali, et } \\
\text { al. [20] }\end{array}$ & Investigator-developed & $\begin{array}{l}\text { Perceived stress scale } \\
\text { (PSS) [80] }\end{array}$ & $\begin{array}{l}\text { Cohen's perceived stress scores were significantly higher ( } p \\
<0.05 \text { ) in students who used their smartphones more than } 2 \\
\text { hours per day }\end{array}$ \\
\hline $\begin{array}{l}\text { Gao, et al. } \\
{[25]}\end{array}$ & $\begin{array}{l}\text { Mobile phone } \\
\text { addiction index (MPAI) } \\
{[57]}\end{array}$ & $\begin{array}{l}\text { Depression anxiety and } \\
\text { stress scale (DASS-21) } \\
{[58]}\end{array}$ & $\begin{array}{l}\text { In the regression model, stress significantly affected mobile } \\
\text { phone addiction }(B=0.462, t=17.285, p<0.01)\end{array}$ \\
\hline $\begin{array}{l}\text { Kim, et al. } \\
\text { [29] }\end{array}$ & $\begin{array}{l}\text { Smartphone addiction } \\
\text { proneness Scale } \\
\text { (SAPS) [59] }\end{array}$ & Investigator-developed & $\begin{array}{l}\text { Perceived psychological health was closely related to } \\
\text { smartphone overuse }(p<0.05) \text {. Stressed participants made } \\
\text { up a higher percent of the cell phone overuse group }(61.7 \%) \\
\text { and were } 2.2 \text { times more likely to overuse smartphones (OR } \\
2.19 \text {, } \\
95 \% \mathrm{Cl} 1.55-3.10) \text {. Stress was significantly associated with } \\
\text { disturbance of adaptive functions, virtual life orientation, } \\
\text { withdrawal, and tolerance (all } p<0.05) \text {. }\end{array}$ \\
\hline $\begin{array}{l}\text { Kuang- } \\
\text { Tsan, et al. } \\
{[30]}\end{array}$ & $\begin{array}{l}\text { Problematic } \\
\text { cellular phone } \\
\text { use questionnaire } \\
\text { (PCPUQ) [62] }\end{array}$ & $\begin{array}{l}\text { Scale of university } \\
\text { students' daily life stress } \\
\text { [81] }\end{array}$ & $\begin{array}{l}\text { Love affair stress had a strong correlation to mobile phone } \\
\text { addiction }(p<0.01) \text {. Academic stress also showed a } \\
\text { significant correlation }(p<0.05) \text { to mobile phone addiction. }\end{array}$ \\
\hline $\begin{array}{l}\text { Long, et al. } \\
\text { [33] }\end{array}$ & $\begin{array}{l}\text { Problematic cellular } \\
\text { phone } \\
\text { Use questionnaire } \\
\text { (PCPUQ) [62] }\end{array}$ & $\begin{array}{l}\text { Perceived stress scale } \\
\text { (PSS) [80] }\end{array}$ & $\begin{array}{l}\text { Stress scores were significantly higher in students reporting } \\
\text { problematic smartphone use }(z=-6.78, p<0.001) \text {. }\end{array}$ \\
\hline $\begin{array}{l}\text { Samaha, et } \\
\text { al. [40] }\end{array}$ & $\begin{array}{l}\text { Smartphone addiction } \\
\text { scale for college } \\
\text { students (SAS- C) [82] }\end{array}$ & $\begin{array}{l}\text { Perceived stress scale } \\
\text { (PSS) [80] }\end{array}$ & $\begin{array}{l}\text { A significant positive correlation was found between risk of } \\
\text { smartphone addiction and perceived stress }(r=0.193, p \\
<0.01) \text {, with high risk of smartphone addiction associated } \\
\text { with high levels of perceived stress. A negative correlation } \\
\text { was found between risk of smartphone addiction and life } \\
\text { satisfaction }(r=-0.492, p<0.01) \text {. Using linear regression, } \\
\text { risk of smartphone addiction explained } 3.8 \% \text { of the variance } \\
\text { in perceived stress, [F }(3,215)=2.80, p<0.05] \text {. Perceived } \\
\text { stress explained } 24.3 \% \text { of the variance in life satisfaction, } \\
\text { after controlling for sex and age }(p<0.01) \text {. }\end{array}$ \\
\hline $\begin{array}{l}\text { Wang, et } \\
\text { al. [44] }\end{array}$ & $\begin{array}{l}\text { Mobile phone } \\
\text { addiction scale } \\
\text { (MPAS) [73] }\end{array}$ & $\begin{array}{l}\text { Perceived stress scale } \\
\text { (PSS) [80] }\end{array}$ & $\begin{array}{l}\text { Level of perceived stress significantly correlated with } \\
\text { degree of smartphone addiction in both the problematic } \\
\text { smartphone use group }(r=0.33, p<0.01) \text { and the non- } \\
\text { problematic smartphone use group }(r=0.30, p<0.01) \text {. A } \\
\text { multiple regression model showed a significant relationship } \\
\text { between stress and problematic smartphone use }(B=0.31 \text {, } \\
t=5.45, p=0.01) \text {. Perceived stress was also found to } \\
\text { moderate the relationship between entertainment motivation } \\
\text { and problematic smartphone use, and between escapism } \\
\text { motivation and problematic smartphone use among those } \\
\text { scoring highest on problematic use. }\end{array}$ \\
\hline
\end{tabular}


studies, respectively. The Self-Rating Questionnaire for Adolescent Problematic Mobile Phone Use (SQAPMPU) from Tao, et al. [67] and the Mobile Phone Problem Use Scale (PMPU) from Bianchi and Phillips [65] were used in two studies each. The Hong, et al. Mobile Phone Addiction Scale (MPAS-H) [73] and Kim, et al. Smartphone Addiction Proneness Scale (SAPS) [83] were also used in two of the studies in our analysis. It should be noted that the studies assessing mental health sequelae of smartphone use and overuse included in this analysis were conducted in a variety of countries around the world and many of the instruments were translated into the country's specific language prior to use. Validity and reliability assessments of the specific instrument (with or without translation) were sometimes provided by the authors in the individual studies. However, a comprehensive analysis of the validity of the smartphone use scales employed by the studies analyzed was outside the scope of this analysis.

Kwon's SAS is a 33-item self-reporting instrument consisting of six factors: Daily-life disturbance, positive anticipation, withdrawal, cyberspace-oriented relationship, overuse, and tolerance [53]. Each statement is evaluated by respondents on a scale of 1-6, and overall scores can range 33-198, with higher scores corresponding to a higher level of smartphone addiction [53]. The SAS was used by four studies in our analysis [19,21,27,34]. A short version of the SAS (SAS-SV) [51] developed by the same research team was used by three studies in our analysis $[18,22,40]$. The SAS- SV contains 10 items which are evaluated by respondents on the same six-point Likert-type scale as the SAS. Scores on the SAS-SV can range 10-60, with higher scores signifying higher levels of smartphone addiction [51].

The PMPU is a 27-item instrument consisting of statements that respondents evaluate on a 10-point Likert scale from "not true at all" to "extremely true [65]". Scores on the PMPU can range 27-270, which higher scores indicating increasing levels of problematic mobile phone use. Two studies in our analysis used the PMPU $[39,41]$.

Hong, et al. MPAS-H is an 11-item instrument evaluating three factors of smartphone addiction: Time management and its problems, academic problems and influence of smartphones, and using smartphones as a substitute for reality [73]. Respondents evaluate each item on a six-point Likert scale ranging from 1 ("incompletely agree") to 6 ("completely agree"). Higher scores are indicative of higher levels of smartphone addiction. Two studies in our analysis used the MPAS- $H[26,30]$.

Kim, et al. SAPS is a 15-item instrument consisting of statements that respondents evaluate on a four-point Likert scale from 1 ("strongly disagree") to 4 ("strongly agree") [83]. Scores on the SAPS range from 15 to 60 , with higher scores signifying a greater degree of smar- tphone addiction proneness. The SAPS is structured around four domains including disturbance of adaptive functions, withdrawal, tolerance, and virtual life orientation. Two studies in our analysis used the SAPS $[28,29]$.

\section{Smartphone use and anxiety}

Anxiety was a common mental health aspect found to be associated with smartphone use in this scoping review with 19 of the 31 articles (61.3\%) assessing some aspect of anxiety (Table 2). The anxiety construct was assessed via a variety of self-report instruments both as a dependent and an independent variable regarding the relationship with smartphone use. The Beck Anxiety Inventory (BAI) [54], Spielberger's State-Trait Anxiety Inventory (STAI) [48], and Zung's Self-Rating Anxiety Scale (SAS-Z) [52] were the primary instruments and were used in four studies each. Thus, 12 of the 19 studies (63.2\%) analyzing the effect of smartphone use and overuse on anxiety employed one of these three instruments. The remaining seven studies $(36.8 \%)$ each used a different scale to assess anxiety. A full description of the three most commonly used anxiety inventories is provided below, although full psychometric and reliability analyses of all instruments used are outside of the scope of this study. References for the anxiety instruments used by the studies in our analysis are available in Table 1.

The BAI contains 21 statements representing physical and psychological symptoms of anxiety that respondents rate on a scale of "Not at all" (0 points) to "Severely-it bothered me a lot" (3 points) [54]. Scores on the BAI range from 0 to 63 with validated cut offs for "low", "moderate" and "potentially concerning" levels of anxiety. The BAI was used in four studies in this analysis $[21,27,32,38]$.

The STAI is a 40-item instrument divided into two main sections, one intended to assess the "state" of anxiety (ie, the reaction occurring at a given time at a certain intensity; form $\mathrm{Y}-1$ ) and the other intended to assess the "trait" of anxiety (ie, relatively stable proneness to anxiety; form Y-2) [48]. Respondents rate each of the 40 statements on a scale of how well they represent their self-perception ("not at all" [1 point] to "very much so" [4 points]). Scores on each of the sections range from 20 to 80 with higher scores signifying greater degrees of anxiety. The STAI was used in four studies in our review $[16,19,34,41]$.

The SAS-Z is composed of 20 statements related to symptoms of anxiety, each rated by respondents on a scale of "none or a little of the time" to "most or all of the time" [52]. Example statements include "I feel more nervous and anxious than usual" and "I can feel my heart beating fast" [52]. Scores on the SAS-Z range 20-80 with higher scores signifying a greater degree of anxiety symptoms. The SAS-Z was used in four studies in our review $[18,31,33,42]$. 
Anxiety was positively correlated with either problematic or high smartphone use in eight studies $[18,21,23,29,32,33,35,38]$. Hawi and Samaha's study results indicated that students with smartphone addiction showed higher odds of having high anxiety $(O R=$ 4.71, $p=0.008$ ) [27]. Lee, et al. found that with each one-point increase in smartphone dependency score, the risk of abnormal anxiety in men and women increased by $10.1 \%$ and $9.2 \%$, respectively ( $p<0.001$ ) [31]. Chen, et al. study revealed that mobile phone addiction level is a positive significant predictor of negative emotions, including anxiety [17]. Mok, et al. study showed that anxiety levels increased with smartphone addiction levels ( $p<0.001$ ) [34]. A regression analysis performed by Tao, et al. in a study of 4747 Chinese college students found that problematic mobile phone use was an independent predictor of anxiety symptoms (OR 2.02, 95\% Cl 1.68-2.43) [42]. Five studies examined the effects that anxiety has on smartphone use and all showed that anxiety predicted smartphone addiction $[19,25,28,41,84]$. Finally, Cheever, et al. examined the effects that removal of possession of smartphone had on anxiety levels over time. Results indicated that students classified as heavy smartphone users experienced statistically significant increases in overall anxiety ( $p=$ 0.017), as opposed to lighter users of smartphones [16].

\section{Smartphone use and depression}

Depression was the other most common mental health aspect explored by the studies in our analysis. Nineteen of the 31 studies (61.3\%) investigated the consequence of smartphone use on depression outcomes or the effect of depression on smartphone use, as shown in Table 3. Six different scales were used to assess depression in these studies.

The Center for Epidemiologic Studies Depression Scale (CES-D) was employed in five of the studies in our analysis $[17,18,28,36,42]$, which made it the most commonly used depression scale. The CES-D contains 20 statements that center around perceived symptoms of depression, with some statements positively worded (ie, "I enjoyed life") and others negatively worded ("I had crying spells") [69]. Statements are scored on a scale of 0 ("rarely or none of the time [less than one day a week]") to 3 ("most or all of the time [5-7 days a week]"). Positive statements are scored in reverse from negative statements on the same scale. Scores on the CES-D range 0-60 with higher scores indicating a greater degree of depression symptoms [69].

The Beck Depression Inventory-I (BDI-I) is composed of 21 sets of statements that represent varying levels of severity of certain characteristics of depression around four indices: appearance, thought content, vegetative signs, and psychosocial performance [70]. Respondents choose the statement out of four (with a corresponding point value) that best represents their self- perception of their symptoms. An example set of phrases includes,
"I do not feel sad" (0 points), "I feel sad" (1 point), "I am sad all the time and I can't snap out of it" (2 points), "I am so sad and unhappy that I can't stand it" (3 points). Scores range 0-63 with higher scores indicating more severe symptoms. Four studies in our analysis used the BDI-I $[19,21,34,38]$.

Another iteration of the BDI-I was developed by the same research team in the 1990s to more closely align with significant changes to the depression diagnosis and screening guidelines that had occurred since the BDI was first published in the 1960s. The Beck Depression Inventory-II (BDI-II) is composed of 21 items (similar to the BDI-I) covering a range of domains including pessimism, past failure, guilt feelings, and self-dislike, among other attributes [74]. Also like the BDI-I, the BDI-II contains sets of statements from which respondents select one that most closely aligns with their self-perception. Scoring is identical to the BDI-I (0-63), and the BDI-II also has score cut offs for mild, borderline, moderate, severe, and extreme symptoms of depression [74]. The BDI-II was used in one of the studies in our analysis [26].

The Zung Self-Rating Depression Scale (SDS) is composed of 20 statements related to symptoms of depression, each rated by respondents on a scale of "a little of the time" to "most of the time". Example statements include "I feel down-hearted and blue" and "I feel hopeful about the future" [72]. Scores on the SDS range 20-80 with higher scores signifying a greater degree of depression symptoms. The SDS was used in three studies in our analysis $[24,33,43]$.

The Patient Health Questionnaire-9 (PHQ-9) is a nine-item self-report instrument which involves the respondent indicating a level of frequency of various symptoms of depression over the preceding two weeks including "feeling down, depressed, or hopeless" and "poor appetite or overeating" [68]. Scores on the PHQ-9 range from 0 to 27, with higher scores indicating more severe depression symptoms and cut offs for minimal, mild, moderate, moderately severe, and severe depression. The PHQ-9 was used by two of the studies in our analysis $[15,22]$.

The General Health Questionnaire-28 (GHQ-28) is a 28-item self-report instrument that is a shortened version of the larger, more comprehensive 60-item General Health Questionnaire more suited for use in a primary care setting [56]. The GHQ-28 is used to screen for a variety of psychological and psychiatric conditions and consists of four subscales: Somatic symptoms, anxiety and insomnia, social dysfunction, and severe depression. Respondents answer questions related to frequency of symptoms on a four-point Likert scale (0-3 points each), and scores range from 0 to 84 with higher scores corresponding to higher levels of distress. A score of 23 or lower would be classified as non-psychiatric, while scores of 24 or higher would indicate need for psychiatric care [56]. The GHQ-28 was used by one study in our 
analysis [23]; however, the results will be reported separately in the anxiety and depression sections of the tables and manuscript.

Regarding the links between smartphone usage and depression, researchers have studied a variety of different factors. Panova, et al. revealed a correlation between maladaptive mobile phone use and depression $(r=0.194, p<0.01)$ [35]. Likewise, Tao, et al. found that depression symptoms were higher in students with problematic mobile phone use $(p<0.001)$ [42]. Results from Kim, et al. showed that perceived psychological health (which includes depression) is closely related to smartphone overuse $(p<0.05)$. Saadat found positive correlations between mobile phone dependency and depression [38]. Similarly, Demirci found a positive correlation between smartphone addiction and depression [21]. Results from Gao, et al. showed that smartphone addiction and depression were correlated $(p<0.05)$ and that smartphone addiction negatively affected quality of life through the mediating effect of depression [26]. Chen's study of 1441 Chinese students revealed a significant association between smartphone addiction and depression in females, but not males [18]. Two studies in our analysis did not find a link between smartphone use and depression. First, results from Ezoe, et al. study did not indicate a significant correlation between mobile phone dependence and depression $(r=0.12, p>0.05)$ [24]. Second, Mok, et al. latent class analysis showed that smartphone addiction was not a significant factor in differentiating depression levels $(F=0.275, p=0.76)$. Furthermore, one study by Choi, et al. found that higher depression scores were associated with lower levels of smartphone addiction $(p<0.001)$ [19].

Similarly, Elhai, et al. study that used an app to objectively track smartphone usage found that higher baseline depression scores were associated with lower smartphone activity and vice versa [22].

Other researchers examined the predictive nature of smartphone use on depression. Chen, et al. found in a sample of 1089 Chinese college students that level of smartphone addiction was a significant predictor of depression ( $B=0.43, t=3.15, p<0.01)$ and that $45 \%$ of the variation in depression scores was accounted for by the smartphone addiction level in a regression model with good fit $(F[1,40]=7.26, p<0.001)[17]$. A regression analysis performed by Tao, et al. in a study of 4747 Chinese college students found that problematic mobile phone use was an independent predictor of depressive symptoms (OR $=2.53,95 \% \mathrm{Cl} 2.10-3.05)$ [42]. Eyvazlou, et al. study results showed that cellphone overuse was significantly correlated with depression $(p<0.001)$ and a multivariate regression indicated that overuse also predicted higher levels of depression $(p<0.001)$ [23]. Several other researchers examined the effect of depression on smartphone addiction and found that depression was a positive predictor of either smartphone addiction or problematic smartphone use $[15,25,28,33]$. Results from Park, et al. study are a nuanced exception to these findings. They found that participants who used smartphones for bridging weak ties were likely to have lower levels of depression $(p<0.001)$ [36].

\section{Smartphone use and sleep}

Eight of the 31 studies (25.8\%) included in this review analyzed the relationship between sleep and smartphone use (Table 4). The Pittsburgh Sleep Quality Index (PSQI) [77] was used in six of the eight studies (75\%) that evaluated sleep $[18,20,21,23,39,42]$. The PSOI is a self-report instrument that assesses a variety of factors and consists of seven components, all intended to evaluate the quality of sleep over the preceding month prior to administering the survey: Sleep quality, sleep latency, sleep duration, habitual sleep efficiency, sleep disturbances, use of sleeping medications, and daytime dysfunction [77]. The PSQI yields a global score ranging from 0-21, with higher scores indicating worse sleep quality. A score of $\geq 5$ on the PSQI is generally considered indicative of sleep quality issues [77].

The Medical Outcomes Study Sleep Measure [79], used in one of the studies in our analysis [37], is a 12item self-report instrument that evaluates sleep across six indices: 1) Sleep disturbances; 2) Adequacy of sleep; 3) Daytime drowsiness; 4) Snoring; 5) Waking short of breath or with a headache, and 6) Amount of sleep. Respondents rate each of the items based on their perception of the characteristic over the preceding four weeks. Scoring includes a scaled response matrix based on the chosen levels of each index as well as factoring in the quantitative assessment of the amount of sleep of the respondents. Higher scores indicate a greater degree of the particular aspect of sleep being assessed [79]. The final study assessing sleep used an investigator-developed scale [14].

Of the six studies employing the PSQI, four showed a significant positive correlation of PSQI sleep scores with smartphone addiction/problematic use as measured by either the SAS, PMPU, or Cell Phone Overuse Scale (COPS) $[18,21,23,39]$. Eyvazlou, et al. demonstrated a significant correlation between mobile phone overuse and the PSQI global score $(r=0.29, p<0.001)$ [23].

Deepali, et al. study showed that mobile phone use exceeding two hours per day was associated with higher sleep deprivation $(p<0.001)$ [20]. In Tao, et al. study, PMPU and poor sleep quality were observed in $28.1 \%$ and $9.8 \%$ of participants, respectively. Also, a multivariate logistic regression showed independent associations of PMPU and sleep quality with mental health $(p<0.01)$, with a significant interaction between the measures [42]. Rosen, et al. found that daily smartphone usage predicted sleep problems $(p<0.001)$ and participants who checked their phones during sleeping hours experienced more sleep problems $(p<0.001)$, as 
did participants whose phone was placed away from the bed when sleeping $(p<0.05)$ [37]. Alosaimi, et al. reported that $44.1 \%(N=1043)$ participants agreed or strongly agreed that sleeping hours decreased once they started using smartphones [14]. Chen, et al. analyzed smartphone addiction as a predictive factor of sleep quality by sex (ie, male vs. female) in a multivariate regression model and found that poor sleep quality was significantly predictive of smartphone addiction in both sexes (male adjusted OR 3.19 [95\% Cl 2.23-4.58], $\mathrm{p}=$ 0.000; female adjusted OR 2.12 [95\% Cl 1.18-4.51], $\mathrm{p}=$ 0.000) [18].

Demirci, et al. reported that daytime dysfunction (as a component of sleep quality) was higher in the high-use smartphone group compared with the low-use group [21]. Additionally, smartphone use severity was significantly correlated with PSQI global scores $(r=0.156, p=$ 0.014) [21]. Finally, Sahin, et al. analyzed the link between scores on the PMPU and PSQI in a sample of 504 students and found a significant correlation $(r=-0.297 ; p=$ 0.000) [39].

\section{Smartphone use and stress}

Seven of the 31 studies (22.6\%) reviewed pertained to stress and met criteria for inclusion in this review (Table 5). The Perceived Stress Scale (PSS) was used to assess stress in four $(57.1 \%)$ of those studies $[20,33,40,44]$. The PSS is a 14 -item self-report questionnaire using a five-point Likert scale that assesses perceived stress in the last month [80]. The Depression Anxiety and Stress Scale (DASS-21) [58], Experiences in Close Relationships-Revised-Korean [85], and Scale of University Students' Daily Life Stress [86], were used in the remaining three studies.

Stress was found to be a positive predictor of both mobile phone addiction [25] and problematic smartphone use [33]. Kim, et al. reported that study participants who were stressed were 2.2 times more likely to overuse smartphones than those with low stress [29]. Increased use of mobile phones was also associated with higher perceived stress $(p<0.05)$ [20]. Results from Kuang-Tsan, et al. study indicate that academic stress $(p<0.05)$ and love-affair stress $(p<0.001)$ were positively related to smartphone addiction. Interpersonal relationship stress, family life stress, and self-career stress were not significantly related to smartphone addiction [30]. Wang, et al. reported that perceived stress moderates the relationship between entertainment motivation (ie, use smartphones for fun) and problematic smartphone use, as well as between escapism motivation (ie, use smartphones to get away from what one is doing) and problematic smartphone use among those scoring high on problematic use [44]. Finally, Samaha, et al. [40] found a small positive correlation between risk of smartphone addiction and perceived stress $(p<$ 0.002 ), with high risk of smartphone addiction associated with high levels of perceived stress. In linear regres- sion modeling, risk of smartphone addiction explained $3.8 \%$ of the variance in perceived stress [40].

\section{Discussion}

The purpose of this scoping review was to provide and summarize research regarding the association of smartphone use with various parameters of mental health. Overall results of studies in this review strongly suggest that there is a link between smartphone use and mental health related issues of anxiety, depression, and stress. There is also an association with sleep, which may exacerbate some of those issues. However, there are many different nuances in these studies that do not allow one to make definitive statements regarding effects of smartphones on parameters of mental health. Another one of the challenging issues in interpreting studies in this review pertains to the terminology used for smartphone use. Smartphone addiction, problematic smartphone use, and high use of smartphones were the different variables/terms examined in the studies. While it may seem like just a matter of semantics, there are differences in both the philosophy and measurement of those variables. Panova and Carbonell propose moving away from terminology and research referring to "addiction" because of insufficient support to confirm actual addiction [87]. Instead they urge researchers to focus on "problematic use" when studying technology behaviors. To simplify the discussion, we will use the term "problematic use" throughout the remainder of this paper when referring to excessive or high smartphone usage.

\section{Anxiety}

Anxiety and depression were the most common parameters of mental health examined in this review with each appearing in $62.5 \%$ of the studies. This similar to Elhai, et al. finding that depression and anxiety were consistently related to problematic smartphone use [88]. The body of research points to linkages between anxiety and smartphone use; however, one of the difficulties in making strong claims is that 11 different instruments were used to measure anxiety.

Nonetheless, in all nineteen studies anxiety was shown to be either associated with or predictive of problematic smartphone use. Of the mental health attributes examined in this scoping review, studies pertaining to anxiety were not only the most common, but also the ones with most consistent results.

\section{Depression}

The findings regarding depression were perhaps the most interesting. The majority ( $n=14,73.7 \%)$ of studies revealed some type of positive association between depression and problematic smartphone use, but there were five exceptions. Two studies $[24,34]$ found no association, while Choi, et al. and Elhai, et al. found negative correlations between depression and smartphone 
use $[19,22]$. One possible explanation is that those with depression may engage in behavioural avoidance or social isolation, which could extend to digital relationships as well [89].

Results from Park, et al. study revealed a nuance in that the reasons for smartphone usage may also play a factor [34]. When used for bonding purposes, smartphone usage may actually serve as a protective factor against depression. A similar, but alternate explanation posed by Hunter, et al. is that those who are socially excluded or isolated may receive emotional comfort through their phones [90]. Another potential explanation is that problematic smartphone use was measured differently (ie, subjectively versus objectively) in some of the studies, which could lead to different outcomes. All of these findings suggest that the relationship between depression and problematic smartphone use is complex and without a single unifying theme that explains the connection.

\section{Sleep}

While not directly a mental health issue in and of itself, sleep-related problems are common among college students and play an interactive role in student health [91,92]. Sleep-related problems have a negative effect on many aspects of people's lives including cognitive, physical, and mental health [93]. In particular, sleep disturbances are associated with higher rates of depression and anxiety [94]. Although the studies in this review examined different facets of sleep-related problems, all confirm an association between problematic smartphone uses and sleep disturbances. As Tao, et al. noted, one question that needs further study is the degree to which sleep might play a mediating role with regard to problematic smartphone use and aspects of mental health [42]. In other words, if sleep disturbances due to problematic smartphone use can be overcome, will that effectively reduce certain negative mental health outcomes?

\section{Stress}

Extensive research indicates high levels of stress among college students with many different contributing factors [95]. Researchers are still trying to determine the role that smartphones may play in this phenomenon. Some have suggested that smartphones promote an "always on" environment in which users feel compelled to constantly monitor digital communication [96]. In effect, the inability to take a "mental break" from those activities may be creating and/or exacerbating stress levels. In this review, when taken as a whole, studies pertaining to stress present an interesting picture. With stress being both a predictor and outcome of PSU, it shows that there is no clear answer to the direction of the influence. As discussed later in this section, these results may suggest a potential reciprocal effect that needs to be studied further.

\section{Implications}

Although results of studies in this review point toward a connection between problematic smartphone use and mental health, it is difficult to make definitive statements regarding the links. First, the studies are primarily correlational, therefore it is not completely clear which direction the influence flows. While most reported on the influence of smartphone use on the various mental health issues, it is possible that the reverse could be true. Although more difficult to conduct, experimental research is needed to determine cause and effect. It is also possible as Pourrazavi, et al. suggest that there are reciprocal interactions in which mental health issues drive students to smartphone use for comfort or distraction and the overuse exacerbates the issues [97].

Similarly, Brand, et al. have proposed a theoretical framework model (I-PACE) from which to examine technology use disorders. This model suggests potential interactions between personal attributes, affective and cognitive responses to situational triggers, and executive functioning [98]. Second, the cross-sectional nature of the studies also presents a challenge to concluding that problematic smartphone use contributes to negative mental health outcomes. Longitudinal research is needed across all the different mental health issues included in this study. Third, as Ellis and colleagues conclude, self-report instruments may not accurately reflect the true nature of smartphone use [99]. Now that smartphone time and usage tracking apps are readily available, future research should use that actual data in lieu of or in addition to self-report instruments. Fourth, because of the various ways that problematic smartphone use and the mental health issues covered in this review are framed, defined, and measured, it is difficult to make strong comparisons among the studies. It is beyond the scope of this study to delve into those differences, but the findings from this review regarding measurement instruments provide important background information.

There are several practical implications of the findings from this review. First, college mental health counselors should be aware of the association between smartphones and mental health and consider discussing with students in their care as applicable. However, they also need to interpret results of studies linking smartphones and mental health with the methodological issues in mind. Second, researchers need to recognize and address the gaps in the literature, particularly as it applies to lack of experimental studies and longitudinal research. Significant research that addresses the nuances of smartphone use and mental health is needed to provide a clearer picture of those associations.

\section{Limitations}

There are limitations with regard to interpreting the results of this scoping review. Unless noted, all instru- 
ments in this analysis were self-report in nature, meaning the respondent completed the instrument without direct guidance or input from a clinician or investigator. There can be inherent bias in self-reported data, even from an instrument with validity and reliability data related to use in a specific population $[100,101]$. While self-reported information has its benefits, including the ability to obtain direct (from the patient) rather than indirect (through a third party) information, different respondents may interpret the exact same statement or prompt on an instrument in a variety of ways, leading to potential issues in interpreting the results. While notable for an individual study, given the cross-sectional and comprehensive nature of our analysis, interpretation of any effect that self-report bias may have had on outcomes was outside the scope of our analysis.

\section{Conclusion}

The proliferation of smartphone use among college students has coincided with growing mental health concerns. This scoping review provides the current landscape with regard to the association of smartphone use with anxiety, depression, stress, and sleep. Anxiety and depression were the most common mental health parameters studied, with anxiety being the most consistent association with problematic smartphone use. Results from studies on depression revealed mixed results that may be due primarily to differences in measurement. While all the studies in this review showed some form of association between problematic smartphone use with one or more of those mental-health related issues, a number of methodological issues prevent definitive statements regarding smartphones and mental health. Mental health professionals, college counselors, and students should be aware of those associations, and researchers need to conduct studies that eliminate the methodological concerns.

\section{Acknowledgements}

The authors thank Mr. Frank Davis of the University of Kentucky Medical Center Library for his assistance with the literature search strategy and citation retrieval.

\section{References}

1. Center PR (2019) Mobile fact sheet.

2. Anderson MS (2019) The advantages of the smartphone, Techwalla.

3. Katz-Sidlow RJ, Ludwig A, Miller S, Sidlow R (2012) Smartphone use during inpatient attending rounds: Prevalence, patterns and potential for distraction. J Hosp Med 7: 595599.

4. Derks D, Bakker AB (2014) Smartphone use, work-home interference, and burnout: A diary study on the role of recovery. Applied Psychol 63: 411-440.

5. Klauer SG, Guo F, Simons-Morton BG, Ouimet MC, Lee SE, et al. (2014) Distracted driving and risk of road crashes among novice and experienced drivers. N Engl J Med 370: 54-59.
6. Tossell CC, Kortum P, Shepard C, Rahmati A, Zhong L (2015) You can lead a horse to water but you cannot make him learn: Smartphone use in higher education. Brit J Educ Technol 46: 713-724.

7. Twenge JM (2017) IGen: Why today's super-connected kids are growing up less rebellious, more tolerant, less happy-and completely unprepared for adulthood-and what that means for the rest of us. Atria Books, New York.

8. Cain J (2018) It's time to confront student mental health issues associated with smartphones and social media. Am J Pharm Educ 82: 6862.

9. Harris T (2020) How technology is hijacking your mind: From a magician and Google design ethicist.

10. Peters MD, Godfrey CM, Khalil H, Mclnerney P, Parker D, et al. (2015) Guidance for conducting systematic scoping reviews. Int J Evid Based Healthc 13: 141-146.

11. Arksey H, O'Malley L (2005) Scoping studies: Towards a methodological framework. Int J Soc Res Methodol 8: 19-32.

12. Moher D, Shamseer L, Clarke M, Davina G, Alessandro L, et al. (2015) Preferred reporting items for systematic review and meta-analysis protocols (PRISMA-P) 2015 statement. Systematic Rev 4: 1.

13. Ouzzani M, Hammady $H$, Fedorowicz Z, Elmagarmid A (2016) Rayyan: A web and mobile app for systematic reviews. Systematic Rev 5: 210.

14. Alosaimi FD, Alyahya $H$, Alshahwan $H$, Al Mahyijari $N$, Shaik SA (2016) Smartphone addiction among university students in Riyadh, Saudi Arabia. Saudi Med J 37: 675-683.

15. Boumosleh JM, Jaalouk D (2017) Depression, anxiety, and smartphone addiction in university students-A cross sectional study. PLoS One 12.

16. Cheever NA, Rosen LD, Carrier LM, Chavez A (2014) Out of sight is not out of mind: The impact of restricting wireless mobile device use on anxiety levels among low, moderate and high users. Comput Human Behav 37: 290-297.

17. Chen L, Yan Z, Tang WJ, Yang FY, Xie XD, et al. (2016) Mobile phone addiction levels and negative emotions among Chinese young adults: The mediating role of interpersonal problems. Comput Human Behav 55: 856-866.

18. Chen B, Liu F, Ding S, Ying X, Wang L, et al. (2017) Gender differences in factors associated with smartphone addiction: A cross-sectional study among medical college students. BMC Psychiatry 17: 341.

19. Choi SW, Kim DJ, Choi JS, Heejune A, Eun-Jeung C, et al. (2015) Comparison of risk and protective factors associated with smartphone addiction and internet addiction. $\mathrm{J}$ Behav Addict 4: 308-314.

20. Deepali A, Shobha MV, Reddy PS (2015) A study of mobile phone usage on sleep and stress among first year medical students. Res J Pharm Biol Chem Sci 6: 720-723.

21. Demirci K, Akgonul M, Akpinar A (2015) Relationship of smartphone use severity with sleep quality, depression, and anxiety in university students. J Behav Addict 4: 85-92.

22. Elhai JD, Tiamiyu MF, Weeks JW, Levine JC, Picard KJ, et al. (2016) Depression and emotion regulation predict objective smartphone use measured over one week. Pers Individ Dif 133: 21-28.

23. Eyvazlou M, Zarei E, Rahimi A, Abazari M (2016) Association between overuse of mobile phones on quality of sleep and general health among occupational health and safety students. Chronobiol Int 33: 293-300. 
24. Ezoe S, Toda M, Yoshimura K, Naritomi A, Den R, et al. (2009) Relationships of personality and lifestyle with mobile phone dependence among female nursing students. Soc Behav Pers 37: 231-238.

25. Tingting Gao, Jiaomeng $\mathrm{Li}$, Han Zhang, Jinglei Gao, Yixi Kong, et al. (2018) The influence of alexithymia on mobile phone addiction: The role of depression, anxiety and stress. J Affect Disord 225: 761-766.

26. Gao T, Xiang YT, Zhang H, Zhang Z, Mei S (2017) Neuroticism and quality of life: Multiple mediating effects of smartphone addiction and depression. Psych Res 258: 457-461.

27. Hawi NS, Samaha M (2017) Relationships among smartphone addiction, anxiety, and family relations. Behav Inf Technol 36: 1046-1052.

28. Kim E, Cho I, Kim EJ (2017) Structural equation model of smartphone addiction based on adult attachment theory: Mediating effects of loneliness and depression. Asian Nurs Res 11: 92-97.

29. Kim HJ, Min JY, Kim HJ, Min KB (2017) Association between psychological and self-assessed health status and smartphone overuse among Korean college students. J Ment Health 28: 11-16.

30. Kuang-Tsan C, Fu-Yuan H (2017) Study on relationship among university students' life stress, smart mobile phone addiction, and life satisfaction. J Adult Dev 24: 109-118.

31. Lee KE, Kim SH, Ha TY, Young MY, Jai JH, et al. (2016) Dependency on smartphone use and its association with anxiety in Korea. Public Health Rep 131: 411-419.

32. Lepp A, Barkley JE, Karpinski AC (2014) The relationship between cell phone use, academic performance, anxiety, and Satisfaction with Life in college students. Comput Human Behav 31: 343-350.

33. Jiang Long, Tie-Qiao Liu, Yan-Hui Liao, Chang Qi, Hao-Yu $\mathrm{He}$, et al. (2016) Prevalence and correlates of problematic smartphone use in a large random sample of Chinese undergraduates. BMC Psych 16: 408.

34. Jung-Yeon Mok, Sam-Wook Choi, Dai-Jin Kim, Jung-Seok Choi, Jaewon Lee, et al. (2014) Latent class analysis on internet and smartphone addiction in college students. Neuropsychiatr Dis Treat 10: 817-828.

35. Panova T, Lleras A (2016) Avoidance or boredom: Negative mental health outcomes associated with use of information and communication technologies depend on users' motivations. Comput Human Behav 58: 249-258.

36. Park N, Lee H (2012) Social implications of smartphone use: Korean college students' smartphone use and psychological well-being. Cyberpsychol Behav Soc Netw 15: 491497.

37. Rosen L, Carrier LM, Miller A, Rokkum J, Ruiz A (2016) Sleeping with technology: Cognitive, affective, and technology usage predictors of sleep problems among college students. Sleep Health 2: 49-56.

38. Saadat P, Kheyrkhah F, Shafaee SF (2012) Relationship between mobile phone dependency, anxiety, depression and somatic complaints among students of Babol Universities (2012). Int J Adv Biotechnol Res 8: 391-397.

39. Sahin S, Ozdemir K, Unsal A, Temiz N (2013) Evaluation of mobile phone addiction level and sleep quality in university students. Pakistan J Med Sci 29: 913-918.

40. Samaha M, Hawi NS (2016) Relationships among smartphone addiction, stress, academic performance, and satisfaction with life. Comput Human Behav 57: 321-325.
41. Sapacz M, Rockman G, Clark J (2016) Are we addicted to our cell phones? Comput Human Behav 57: 153-159.

42. Tao S, Wu X, Zhang Y, Zhang S, Tong S, et al. (2017) Effects of sleep quality on the association between problematic mobile phone use and mental health symptoms in Chinese college students. Int J Environ Res Public Health 14: 185.

43. Tao SM, Wu XY, Zhang SC, Tong SL, Hao JH, et al. (2017) Association of alcohol use with problematic mobile phone use and depressive symptoms among college students in Anhui, China. J Pub Health-Heidelberg 25: 103-112.

44. Wang JL, Wang HZ, Gaskin J, Wang LH (2015) The role of stress and motivation in problematic smartphone use among college students. Comput Human Behav 53: 181188.

45. Lin YH, Chang LR, Lee YH, Tseng HW, Kuo TB, et al. (2014) Development and validation of the smartphone addiction inventory (SPAI). PLoS One 9: e98312.

46. Spitzer RL, Kroenke K, Williams JB, Löwe B (2006) A brief measure for assessing generalized anxiety disorder: The GAD-7. Arch Intern Med 166: 1092-1097.

47. Rosen LD, Whaling K, Carrier LM, Cheever NA, Rokkum $\mathrm{J}$ (2013) The media and technology usage and attitudes scale: An empirical investigation. Comput Human Behav 29: 2501-2511.

48. Spielberger CD (1983) Manual for the state-trait anxiety inventory: STAI (Form Y). Palo Alto, CA: Consulting psychologists press.

49. Xiong J, Zhou Z, Chen W, You Z, Zhai Z (2012) Development of the mobile phone addiction tendency scale for college students. Chin Ment Health J 26: 222-225.

50. Fenigstein A, Scheier MF, Buss AH (1975) Public and private self-consciousness: Assessment and theory. J Consult Clin Psychol 43: 522-527.

51. Kwon M, Kim DJ, Cho H, Yang S (2013) The smartphone addiction scale: Development and validation of a short version for adolescents. PLoS One 8: e83558.

52. Zung WW (1971) A rating instrument for anxiety disorders. Psychosomatics 12: 371-379.

53. Min Kwon, Joon-Yeop Lee, Wang-Youn Won, Jae-Woo Park, Jung-Ah Min, et al. (2013) Development and validation of a smartphone addiction scale (SAS). PLoS One 8: e56936.

54. Beck AT, Epstein N, Brown G, Steer RA (1988) An inventory for measuring clinical anxiety: Psychometric properties. J Consult Clin Psychol 56: 893-897.

55. Jenaro C, Flores N, Gomez-Vela M, Gonzalez-Gil F, Caballo C (2007) Problematic internet and cell-phone use: Psychological, behavioral, and health correlates. Addict Res Theor 15: 309-320.

56. Goldberg DP, Hillier VF (1979) A scaled version of the general health questionnaire. Psychol Med 9: 139-145.

57. Leung $L$ (2008) Linking psychological attributes to addiction and improper use of the mobile phone among adolescents in Hong Kong. J Child Media 2: 93-113.

58. Gong X, Xie XY, Xu R, Luo YJ (2010) Psychometric properties of the Chinese versions of DASS-21 in Chinese college students. Chin J Clin Psychol 18: 443-446.

59. Shin K, Kim D, Jung Y (2011) Development of Korean smart phone addiction proneness scale for youth and adults. Seoul: Korean National Information Society Agency. 
60. Brennan KA, Clark CL, Shaver PR (1998) Self-report measurement of adult attachment: An integrative overview.

61. Yang S (2002) A study of mobile phone addiction of adolescent in high school students. Seoul: Korea Institute for Youth Deve.

62. Yen CF, Tang TC, Yen JY, Huang CL, Chi FH, et al. (2009) Symptoms of problematic cellular phone use, functional impairment and its association with depression among adolescents in Southern Taiwan. J Adolesc 32: 863-873.

63. Beranuy Fargues M, Chamarro Lusar A, Graner Jordania C, Carbonell Sanchez X (2009) Validation of two brief scales for Internet addiction and mobile phone problem use. Psicothema 21: 480-485.

64. Watson D, Weber K, Assenheimer JS, Clark LA, Strauss ME, et al. (1995) Testing a tripartite model: I. Evaluating the convergent and discriminant validity of anxiety and depression symptom scales. J Abnorm Psychol 104: 3-14.

65. Bianchi A, Phillips JG (2005) Psychological predictors of problem mobile phone use. Cyberpsychol Behav 8: 39-51.

66. Wong QJ, Moulds ML (2011) A new measure of the maladaptive self-beliefs in social anxiety: Psychometric properties in a non-clinical sample. J Psychopathol Behav Assess 33: $273-284$

67. Tao S, Fu J, Wang H, Hao J, Tao F (2013) The development of self-rating questionnaire for adolescent problematic mobile phone use and the psychometric evaluation in undergraduates. Chin J Sch Health 34: 26-29.

68. Kroenke K, Spitzer R, Williams W (2001) The patient health questionnaire (PHQ-9)-overview. J Gen Int Med 16: 606613.

69. Radloff LS (1977) The CES-D scale: A self-report depression scale for research in the general population. Appl Psychol Meas 1: 385-401.

70. Beck AT, Ward C, Mendelson M, Mock J, Erbaugh J (1961) Beck depression inventory (BDI). Arch Gen Psychiatry 4: 561-571.

71. Toda M, Monden K, Kubo K, Morimoto K (2004) Cellular phone dependence tendency of female university students. Jap J Hyg 59: 383-386.

72. Zung WW (1965) A self-rating depression scale. Arch Gen Psychiatry 12: 63-70.

73. Hong FY, Chiu SI, Huang DH (2012) A model of the relationship between psychological characteristics, mobile phone addiction and use of mobile phones by Taiwanese university female students. Comput Human Behav 28 2152-2159.

74. Beck AT, Steer RA, Ball R, Ranieri WF (1996) Comparison of beck depression inventories-IA and-II in psychiatric outpatients. J Pers Assess 67: 588-597.

75. Beranuy M, Oberst U, Carbonell X, Chamarro A (2009) Problematic internet and mobile phone use and clinical symptoms in college students: The role of emotional intelligence. Comput Human Behav 25: 1182-1187.

76. Merlo LJ, Stone AM, Bibbey A (2013) Measuring problematic mobile phone use: Development and preliminary psychometric properties of the pump scale. J Addict 2013.

77. Buysse DJ, Reynolds CF, Monk TH, Berman SR, Kupfer DJ (1989) The pittsburgh sleepquality index: A new instrument for psychiatric practice and research. Psychiatry Res 28: 193-213.

78. Assaad S, Costanian C, Haddad G, Tannous F (2014) Sle- ep patterns and disorders among university students in Lebanon. J Res Health Sci 14: 198-204.

79. Hays RD, Martin SA, Sesti AM, Spritzer KL (2005) Psychometric properties of the medical outcomes study sleep measure. Sleep Med 6: 41-44.

80. Cohen S, Kamarck T, Mermelstein R (1983) A global measure of perceived stress. J Health Soc Behav 24: 385-396.

81. Renner MJ, Mackin RS (2001) A life stress instrument for classroom use. Teaching Psychology 25.

82. Sun JCY (2014) Influence of polling technologies on student engagement: An analysis of student motivation, academic performance, and brainwave data. Comput Educ 72: 80-89.

83. Kim D, Lee Y, Lee J, Nam JK, Chung Y (2014) Development of Korean smartphone addiction proneness scale for youth. PLoS One 9.

84. Matar Boumosleh J, Jaalouk D (2017) Depression, anxiety, and smartphone addiction in university students- A cross sectional study. PLoS One 12.

85. Kim SH, Sherry AR, Lee YS, Kim CD (2011) Psychometric properties of a translated Korean adult attachment measure. Meas Eval Couns Dev 44: 135-150.

86. Lee CC, Chen CY (2004) Pressure faced and coped by senior students of National Taiwan Normal University and related factors. Chin J School Health 44: 1-31.

87. Panova T, Carbonell $X$ (2018) Is smartphone addiction really an addiction? J Behav Addict 7: 252-259.

88. Elhai JD, Dvorak RD, Levine JC, Hall BJ (2017) Problematic smartphone use: A conceptual overview and systematic review of relations with anxiety and depression psychopathology. J Affect Disord 207: 251-259.

89. De Silva MJ, McKenzie K, Harpham T, Huttly SR (2005) Social capital and mental illness: A systematic review. J Epidemiol Community Health 59: 619-627.

90. Hunter JF, Hooker ED, Rohleder N, Pressman SD (2018) The use of smartphones as a digital security blanket: The influence of phone use and availability on psychological and physiological responses to social exclusion. Psychosom Med 80: 345-352.

91. Orzech KM, Salafsky DB, Hamilton LA (2011) The state of sleep among college students at a large public university. $J$ Am Coll Health 59: 612-619.

92. Lund HG, Reider BD, Whiting AB, Prichard JR (2010) Sleep patterns and predictors of disturbed sleep in a large population of college students. J Adolesc Health 46: 124-132.

93. Strine TW, Chapman DP (2005) Associations of frequent sleep insufficiency with health-related quality of life and health behaviors. Sleep Med 6: 23-27.

94. Anderson KN, Bradley AJ (2013) Sleep disturbance in mental health problems and neurodegenerative disease. Nat Sci Sleep 5: 61.

95. Leppink EW, Odlaug BL, Lust K, Christenson G, Grant JE (2016) The young and the stressed: Stress, impulse control, and health in college students. J Nerv Ment Dis 204: 931-938.

96. Turkle S (2008) Always-on/Always-on-you: The tethered self. James E Katz. Cambridge.

97. Pourrazavi S, Allahverdipour H, Jafarabadi MA, Matlabi $\mathrm{H}$ (2014) A socio-cognitive inquiry of excessive mobile phone use. Asian J Psychiatr 10: 84-89. 
98. Brand M, Young KS, Laier C, Wölfling K, Potenza MN (2016) Integrating psychological and neurobiological considerations regarding the development and maintenance of specific internet-use disorders: An interaction of person-affect-cognition-execution (I-PACE) model. Neurosci Biobehav Rev 71: 252-266.

99. Ellis DA, Davidson BI, Shaw H, Geyer K (2019) Do smartphone usage scales predict behavior? Int J Hum Comput Stud 130: 86-92.
100. Rosenman R, Tennekoon V, Hill LG (2011) Measuring bias in self-reported data. Int J Behav Healthc Res 2: 320332.

101. Althubaiti A (2016) Information bias in health research: Definition, pitfalls, and adjustment methods. J Multidiscip Healthc 9: 211-217. 